\title{
NON-COMPACT RANDOM GENERALIZED GAMES AND RANDOM QUASI-VARIATIONAL INEQUALITIES
}

\author{
XIAN-ZHI YUAN \\ Department of Mathematics, Statistics, and Computing Science \\ Dalhousie University, Halifax, N.S., Canada B3H $3 \mathrm{~J} 5$ \\ and \\ Department of Mathematics \\ The University of Queensland, Brisbane, Australia 4072
}

(Received October, 1993; revised June, 1994)

\begin{abstract}
In this paper, existence theorems of random maximal elements, random equilibria for the random one-person game and random generalized game with a countable number of players are given as applications of random fixed point theorems. By employing existence theorems of random generalized games, we deduce the existence of solutions for non-compact random quasi-variational inequalities. These in turn are used to establish several existence theorems of noncompact generalized random quasi-variational inequalities which are either stochastic versions of known deterministic inequalities or refinements of corresponding results known in the literature.

Key words: Polish Space, Suslin Space, Measurable Space, Suslin Family, (Random) Fixed Point, (Random) Maximal Element, (Random) Equilibria, (Random) Qualitative Game, (Random) Generalized Game, (Random) Variational Inequality, (Random) Quasi-Variational Inequality, Class $L, L$ Majorized, Measurable Selection Theorem, Property $(K)$, Random Operator.
\end{abstract}

AMS (MOS) subject classifications: Primary 47B80, 47H04, 47H10, 47H40, 47N10，49J40，49J45，49J55，54C60，60H25，90A14，90D13，99D15，93E05; Secondary 28A05, 28A20.

\section{Introduction}

Since Spacek [34] and Hans [14] established some existence results of random fixed point theorems in the fifties, random fixed point theory has received much more attention in recent years, e.g., see Bharucha-Reid [5], Bocsan [8], Engl [13], Itoh [16], Kucia and Nowak [21], Lin [23], Liu and Chen [24], Nowak [26], Papageorgiou [27], Rybinski [28], Sarbadhikari and Srivastava [31], Sehgal and Singh [32], Tan and Yuan [37-38] and Xu [45], etc. Recently, we proved a very general random fixed point theorem in [37] (e.g., see Theorem A below). In this paper, as applications of random fixed point theorem in [37], existence theorems of random maximal elements, random equilibria for a random one-person game and random generalized games with a countable number of players are given. By employing existence theorems of random generalized games, we deduce the existence of solutions for non-compact random quasivariational inequalities which in turn are used to establish several existence theorems of noncompact generalized random quasi-variational inequalities which are either stochastic versions or 
improvements of corresponding results in the literature, e.g., Aliprantis et al. [1], Arrow and Debreu [2], Aubin [3], Aubin and Ekeland [4], Bharucha-Reid [5], Borglin and Keiding [6], Border [7], Bocsan [8], Hans [14], Kucia and Nowak [21], Liu and Chen [24], Mas-Colell and Zame [25], Nowak [26], Papageorgiou [27], Rybinski [28], Shih and Tan [33], Spacek [34], Tan [35-36], Tan and Yuan [39], Tarafdar and Mehta [41], Toussaint [42], Tulcea [43], Yannelis and Prabhakar [46], Zhang (Chang) [47], and Zhou and Chen [48].

\section{Preliminaries}

The set of all real numbers is denoted by $\mathbb{R}$ and the set of natural numbers is denoted by $\mathbb{N}$. If $X$ is a set, we shall denote by $2^{X}$ the family of all subsets of $X$. Let $A$ be a subset of a topological space $X$. The set $A$ is said to be compactly open if $A$ is relatively open in each nonempty compact subset of $X$. We shall denote by $i n t_{X}(A)$ the interior of $A$ in $X$ and by $c l_{X}(A)$ the closure of $A$ in $X$. If $A$ is a subset of a vector space, we shall denote by $c o A$ the convex hull of $A$. If $A$ is a non-empty subset of a topological vector space $E$ and $S, T: A \rightarrow 2^{E}$ are correspondences, then $c o T, T \cap S: A \rightarrow 2^{E}$ are correspondences defined by $(\operatorname{coT} T)(x)=\operatorname{coT}(x)$ and $(T \cap S)(x)=T(x) \cap S(x)$ for each $x \in A$. If $X$ and $Y$ are topological spaces and $(\Omega, \Sigma)$ is a measurable space (see definition below), and $T: \Omega \times X \rightarrow 2^{Y}$ is a correspondence, the Graph of $T$, denoted by GraphT, is the set $\{(\omega, x, y) \in \Omega \times X \times Y: y \in T(\omega, x)\}$ and the correspondence $\bar{T}: \Omega \times X \rightarrow 2^{Y}$ is defined by $\bar{T}(\omega, x)=\left\{y \in Y:(x, y) \in c l_{X \times Y} \operatorname{GraphT}(\omega, \cdot)\right\}$, and $\operatorname{clT}: \Omega \times X \rightarrow 2^{Y}$ is defined by $c l T(\omega, x)=c l_{Y}(T(\omega, x))$ for each $(\omega, x) \in \Omega \times X$. It is easy to see that $\operatorname{cl} T(\omega, x) \subset \bar{T}(\omega, x)$ for each $(\omega, x) \in \Omega \times X$.

If $X$ and $Y$ are topological spaces, $A \subset X \times Y$, and $F: X \rightarrow 2^{Y}$, then

(1) the domain of $F$, denoted by $D o m F$, is the set $\{x \in X: F(x) \neq \emptyset\}$;

(2) the projection of $A$ into $X$, denoted by $\operatorname{Proj}_{X} A$, is the set $\{x \in X$ : there exists some $y \in Y$ such that $(x, y) \in A\}$;

(3) $\quad F$ is said to be lower (respectively, upper) semicontinuous if for each closed (respectively, open) subset $C$ of $Y$, the set $\{x \in X: F(x) \subset C\}$ is closed (respectively, open) in $X$;

(4) $\quad F$ is said to be compact if for each $x \in X$, there exists a open neighborhood $V_{x}$ of $x$ in $X$ such that $F\left(V_{x}\right)=\cup_{z \in V_{x}} F(z)$ is relatively compact in $Y$; and

(5) $\quad x \in X$ is a maximal element of $F$ if $F(x)=\emptyset$.

Note that $\operatorname{DomF}=\operatorname{Proj}_{X} G r a p h F$.

Let $X$ be a subset of a topological vector space $E$. The set $X$ is said to have the property $(K)$ (see [43]) if for each compact subset $S$ of $X$, the convex hull $c o B$ of $B$ is relatively compact in $X$.

Let $X$ be a topological space, $Y$ a non-empty subset of a vector space $E, \theta: X \rightarrow E$ a (singlevalued) mapping and $\phi: X \rightarrow 2^{Y}$ a mapping. Then

(1) $\phi$ is said to be of class $L_{\theta}$ if for every $x \in X, \operatorname{co\phi }(x) \subset Y$ and $Q(x) \notin \operatorname{co\phi }(x)$ and for each $y \in Y, \phi^{-1}(y):=\{x \in X: y \in \phi(x)\}$ is compactly open in $X$;

(2) a correspondence $\phi_{x}: X \rightarrow 2^{Y}$ is said to be an $L_{\theta}$-majorant of $\phi$ at $x \in X$ if there exists an open neighborhood $N_{x}$ of $x$ in $X$ such that

(a) for each $z \in N_{x}, \phi(z) \subset \phi_{x}(z)$ and $\theta(z) \notin \operatorname{co} \phi_{x}(z)$,

(b) for each $z \in X, \operatorname{co\phi }_{x}(z) \subset Y$ and

(c) for each $y \in Y, \phi_{x}^{-1}(y)$ is compactly open in $X$;

(3) $\phi$ is $L_{\theta}$-majorized if for each $x \in X$ with $\phi(x) \neq \emptyset$, there exists an $L_{\theta}$-majorant of $\phi$ at $x \in X$.

We shall only deal with either the case $(I) X=Y$ and which is a non-empty convex subset of a 
topological vector space and $\theta=I_{X}$, the identity mapping on $X$ (in this case, the above notions coincide with the corresponding notions introduced in [46]), or the case (II) $X=\Pi_{i \in I} X_{i}$ and $\theta=\pi_{j}: X \rightarrow X_{j}$ is the projection of $X$ onto $X_{j}$ and $X_{j}=Y$ is a non-empty convex subset of atopological vector space. In both cases $(I)$ and $(I I)$, we shall write $L$ in place of $L_{\theta}$.

A measurable space $(\Omega, \Sigma)$ is a pair where $\Omega$ is a set and $\Sigma$ is a $\sigma$-algebra of subsets of $\Omega$. If $X$ is a set, $A \subset X$, and $\mathscr{D}$ is a non-empty family of subsets of $X$, we shall denote by $\mathscr{D} \cap A$ the family $\{D \cap A: D \in \mathscr{D}\}$ and by $\sigma_{X}(\mathscr{D})$ the smallest $\sigma$-algebra on $X$ generated by $\mathscr{D}$. If $X$ is a topological space with topology $\tau_{X}$, we shall use $\mathscr{B}(X)$ to denote $\sigma_{X}\left(\tau_{X}\right)$, the Borel $\sigma$-algebra on $X$. If $(\Omega, \Sigma)$ and $(\Phi, \Gamma)$ are two measurable spaces, then $\Sigma \otimes \Gamma$ denotes the smallest $\sigma$-algebra on $\Omega \times \Phi$ which contains all the sets $A \times B$, where $A \in \Sigma, B \in \Gamma$, i.e., $\Sigma \otimes \Gamma=\sigma_{\Omega \times \Phi}(\Sigma \times \Gamma)$. We note that the Borel $\sigma$-algebra $\mathscr{B}\left(X_{1} \times X_{2}\right)$ contains $\mathscr{B}\left(X_{1}\right) \otimes \mathfrak{B}\left(X_{2}\right)$ in general. A mapping $f: \Omega \rightarrow \Phi$ is said to be $(\Sigma, \Gamma)$ measurable (or simply, measurable) if for each $B \in \Gamma, f^{-1}(B)=\{x \in \Omega: f(x) \in$ $B\} \in \Sigma$. Let $X$ be a topological space and $F:(\Omega, \Sigma) \rightarrow 2^{X}$ be a mapping. Then $F$ is said to be measurable (respectively, weakly measurable)) if $F^{-1}(B)=\{\omega \in \Omega: F(\omega) \cap B \neq \emptyset\} \in \Sigma$ for each closed (respectively, open) subsets $B$ of $X$. The map $F$ is said to have a measurable graph if GraphF: $=\{\omega, y) \in \Omega \times X: y \in F(\omega)\} \in \Sigma \otimes \mathscr{B}(X)$. A function $f: \Omega \rightarrow X$ is a measurable selection of $F$ if $f$ is a measurable function such that $f(\omega) \in F(\omega)$ for all $\omega \in \Omega$.

If $(\Omega, \Sigma)$ and $(\Phi, \Gamma)$ are measurable spaces, $Y$ is a topological space, then a mapping $F: \Omega \times$ $\Phi \rightarrow 2^{Y}$ is called (jointly) measurable (respectively, weakly measurable) if for every closed (respectively, open) subset $B$ of $Y, F^{-1}(B)=\{(\omega, x) \in \Omega \times \Phi: F(\omega, x) \cap B \neq \emptyset\} \in \Sigma \otimes \Gamma$. In the case $\Phi=X$, a topology space, then it is understood that $\Gamma$ is the Borel $\sigma$-algebra $\mathscr{B}(X)$.

A topological space $X$ is

(i) a Polish space if $X$ is separable and metrizable by a complete metric;

(ii) a Suslin space if $X$ is a Hausdorff topological space and the continuous image of a Polish space.

A Suslin subset in a topological space is a subset which is a Suslin space. "Suslin" sets play very important roles in measurable selection theory. We also note that if $X_{1}$ and $X_{2}$ are Suslin spaces, then $\mathfrak{B}\left(X_{1} \times X_{2}\right)=\mathscr{B}\left(X_{1}\right) \times \mathfrak{B}\left(X_{2}\right)$ (e.g., see [29, p. 113]).

Denote by $g$ and $\mathscr{F}$ the sets of infinite and finite sequences of positive integers respectively. Let $\mathcal{G}$ be a family of sets and $F: \mathrm{gl} \rightarrow \mathcal{F}$ be a map. For each $\sigma=\left(\sigma_{i}\right)_{i=1}^{\infty} \in \mathcal{g}$ and $n \in \mathbb{N}$, we shall denote $\left(\sigma_{1}, \ldots, \sigma_{n}\right)$ by $\sigma \mid n$; then $\bigcup_{\sigma \in g} \bigcap_{n=1}^{\infty} F(\sigma \mid n)$ is said to be obtained from $\mathcal{g}$ by the Suslin operation. Now, if every set obtained from $\mathcal{G}$ in this way is also in $\mathcal{G}$, then $\mathcal{G}$ is called a Suslin family (e.g., see [22], [30], [44], etc.).

Let $X$ and $Y$ be topological spaces, $(\Omega, \Sigma)$ a measurable space and $F: \Omega \times X \rightarrow 2^{Y}$ be a mapping. Then

(a) $\quad F$ is a random operator if for each fixed $x \in X$, the mapping $F(\cdot, x): \Omega \rightarrow 2^{Y}$ is a measurable map;

(b) $F$ is random lower semicontinuous (respectively, random upper semicontinuous, random continuous) if $F$ is a random operator and for each fixed $\omega \in \Omega, F(\omega, \cdot)$ : $X \rightarrow 2^{Y}$ is lower semicontinuous (respectively, upper semicontinuous, continuous); and

(c) a measurable (single-valued) mapping $\psi: \Omega \rightarrow X$ is said to be a random maximal element of the correspondence $F$ if $F(\omega, \psi(\omega))=\emptyset$ for all $\omega \in \Omega$.

Let $(\Omega, \Sigma)$ be a measurable space, $X$ a topological space and $F: \Omega \times X \rightarrow 2^{X}$ a mapping. The (single-valued) mapping $\varphi: \Omega \rightarrow X$ is said to be

(i) a deterministic fixed point of $F$ if $\varphi(\omega) \in F(\omega, \varphi(\omega))$ for all $\omega \in \Omega$; and

(ii) a random fixed point of $F$ if $\varphi$ is a measurable mapping and $\varphi(\omega) \in F(\omega, \varphi(x))$ for all $\omega \in \Omega$. 
It should be noted here that some authors define a random fixed point of $F$ to be a measurable mapping $\varphi$ such that $\varphi(\omega) \in F(\omega, \varphi(\omega))$ for almost every $\omega \in \Omega$, e.g., see [27], [28] and the references therein.

Let $I$ be any set of players and $(\Omega, \Sigma)$ be a measurable space. For each $i \in I$, let its strategy set $X_{i}$ be a non-empty subset of a topological vector space. Let $X=\Pi_{i \in I} X_{i}$. For each $i \in I$, let $P_{i}: \Omega \times X \rightarrow 2^{X_{i}}$ be a correspondence. The collection $\Gamma=\left(\Omega, X_{i}, P_{i}\right)_{i \in I}$ will be called a random qualitative game. A measurable map $\psi: \Omega \rightarrow X$ is said to be a random equilibrium of the random qualitative game $\Gamma$ if $P_{i}(\omega, \psi(\omega))=\emptyset$ for all $i \in I$ and all $\omega \in \Omega$.

A random generalized game (abstract economy) is a collection $\Gamma=\left(\Omega ; X_{i} ; A_{i}, B_{i} ; P_{i}\right)_{i \in I}$ where $I$ is a (finite or infinite) set of players (agents) such that for each $i \in I, X_{i}$ is a non-empty subset of a topological vector space and $A_{i}, B_{i}: \Omega \times X \rightarrow 2^{X_{i}}$ are random constraint correspondences where $X=\Pi_{i \in I} X_{i}$, and $P_{i}: \Omega \times X \rightarrow 2 X_{i}$ is a preference correspondence (which are interpreted as for each player (or agent) $i \in I$, the associated constraint and preferences $A_{i}$, $B_{i}$ and $P_{i}$ have stochastic actions). A random equilibrium of $\Gamma$ is a (single-valued) measurable mapping $\Psi: \Omega \rightarrow X$ such that for each $i \in I, \quad \pi_{i}(\psi(\omega)) \in \bar{B}_{i}(\omega, \psi(\omega))$ and $A_{i}(\omega, \psi(\omega)) \cap$ $P_{i}(\omega, \psi(\omega))=\emptyset$ for all $\omega \in \Omega$. Here, $\pi_{i}$ is the projection from $X$ onto $X_{i}$. If $x \in X$, we shall also write $x_{i}$ in place of $\pi_{i}(x)$ if there is no ambiguity. We remark that if $A_{i}, B_{i}$ and $P_{i}$ of the random generalized game $\Gamma=\left(\Omega ; X_{i} ; A_{i}, B_{i} ; P_{i}\right)_{i \in I}$ are independent of the variable $\omega \in \Omega$, i.e., $A_{i}(\omega, \cdot)=A_{i}(\cdot), B_{i}(\omega, \cdot)=B_{i}(\cdot)$ and $P_{i}(\omega, \cdot)=P_{i}(\cdot)$ for all $\omega \in \Omega$, when $\bar{B}_{i}(\widehat{x})=c l_{X} B_{i}(\widehat{x})$ for each $\widehat{x} \in X$ (which is the case when $B_{i}$ has a closed graph in $X \times X_{i}$; in particular, when clB $B_{i}$ is upper semicontinuous with closed values), our definition of an equilibrium point coincides with that of Ding et al. [12] in the deterministic case; and if in addition, $A_{i}=B_{i}$ for each $i \in I$, our definition of an equilibrium point coincides with the standard definition of the deterministic case, e.g., in Borglin and Keiding [7], Tulcea [43], and Yannelis and Prabhakar [46].

We shall now list some results which will be needed in this paper. The following very general random fixed point theorem is Theorem 2.2 of Tan and Yuan in [37].

Theorem A. Let $(\Omega, \Sigma)$ be a measurable space, $\Sigma$ a Suslin family and $X$ a Suslin space. Suppose $F: \Omega \times X \rightarrow 2^{X} \backslash\{\emptyset\}$ is such that Graph $F \in \Sigma \otimes \mathfrak{B}(X \times X)$. Then $F$ has a random fixed point if and only if $F$ has a deterministic fixed point in $X$, i.e., for each $\omega \in \Omega, F(\omega, \cdot)$ has a fixed point in $X$.

For a non-self mapping generalization of the above result, we refer the reader to [38, Theorem 2.3]. The following measurable selection theorem is due to Leese [22, Corollary, p. 408-409].

Theorem B. Let $(\Omega, \Sigma)$ be a measurable space, $\Sigma$ a Suslin family and $X$ a Suslin space. Suppose $F: \Omega \rightarrow 2^{X}$ has non-empty values such that GraphF $\in \Sigma \otimes \mathfrak{B}(X)$. Then there exists a sequence $\left\{g_{n}\right\}_{n=1}^{\infty}$ of measurable selections of $F$ such that for each $\omega \in \Omega$, the set $\left\{g_{n}(\omega): n \in \mathbb{N}\right\}$ is dense in $F(\omega)$.

The following lemma is Theorem 3.3 of Tan and Yuan in [39].

Lemma 1. Let $\mathrm{G}=\left(X_{i} ; A_{i}, B_{i} ; P_{i}\right)_{i \in I}$ be an abstract economy such that $X=\Pi_{i \in I} X_{i}$ is paracompact. Suppose the following conditions are satisfied:

(a) for each $i \in I, X_{i}$ is a non-empty convex subset of a locally convex Hausdorff topological vector space $E_{i}$;

(b) for each $i \in I, A_{i}: X \rightarrow 2^{X}$ is lower semicontinuous such that for each $x \in X, A_{i}(x)$ is non-empty and $\operatorname{co} A_{i}(x) \subseteq B_{i}(x)$;

(c) for each $i \in I, A_{i} \cap P_{i}$ is $L_{C}$-majorized;

(d) for each $i \in I$, the set $E^{i}=\left\{x \in X:\left(A_{i} \cap P_{i}\right)(x) \neq \emptyset\right\}$ is open in $X$;

(e) there exist a non-empty compact convex subset $X_{0}$ of $X$ and a non-empty compact 
subset $K$ of $X$ such that for each $y \in X \backslash K$ there is an $x \in \operatorname{co}\left(X_{0} \cup\{y\}\right)$ with $x_{i} \in$ $\operatorname{co}\left(A_{i}(y) \cap P_{i}(y)\right)$ for all $i \in I$.

Then $g$ has an equilibrium point in $K$, i.e., there exists a point $\widehat{x}=\left(\widehat{x}_{i}\right)_{i \in I} \in K$ such that for each $i \in I, \widehat{x}_{i} \in \overline{B_{i}}(\widehat{x})$ and $A_{i}(\widehat{x}) \cap P_{i}(\widehat{x})=\emptyset$.

The following result is Theorem 5.3 of Tan and Yuan in [40].

Lemma 2. Let $\Gamma=\left(X_{i}, A_{i}, B_{i}, P_{i}\right)_{i \in I}$ be an abstract economy such that $X=\Pi_{i \in I} X_{i}$ is paracompact. Suppose the following conditions are satisfied:

(a) for each $i \in I, X_{i}$ is a non-empty closed convex subset of a locally convex Hausdorff topological vector space $E_{i}$ and $X_{i}$ has the property $(K)$;

(b) for each $i \in I, B_{i}$ is compact and upper semicontinuous with non-empty compact convex values and $A_{i}(x) \subset B_{i}(x)$ for each $x \in X$;

(c) for each $i \in I, P_{i}$ is lower semicontinuous and $L_{C}$-majorized;

(d) for each $i \in I, E^{i}=\left\{x \in X:\left(A_{i} \cap P_{i}\right)(x) \neq \emptyset\right\}$ is open in $X$;

(e) there exist a nonempty compact convex subset $X_{0}$ of $X$ and a non-empty compact subset $K$ of $X$ such that for each $y \in X \backslash K$ there is an $x \in \operatorname{co}\left(X_{0} \cup\{y\}\right)$ with $x_{i} \in$ $\operatorname{co}\left(A_{i}(y) \cap P_{i}(y)\right)$ for all $i \in I$.

Then there exists $\bar{x}\left(\widehat{x}_{i}\right)_{i \in I} \in K$ such that for each $i \in I, \bar{x}_{i} \in \bar{B}_{i}(\bar{x})$ and $A_{i}(\bar{x}) \cap P_{i}(\bar{x})=\emptyset$.

We also need the following result (e.g., see Theorem 1 of Ding and Tan [11]).

Lemma 3. Let $X$ be a non-empty paracompact convex subset of a Hausdorff topological vector space and $P: X \rightarrow 2^{X}$ be L-majorized (i.e., $L_{I_{X}}$-marjorized). Suppose that there exist a non-empty compact convex subset $X_{0}$ of $X$ and a non-empty compact subset $K$ of $X$ such that for each $y \in z \backslash K$, there exists $x \in \operatorname{co}\left(X_{0} \cup\{y\}\right)$ with $x \in \operatorname{coP}(y)$. Then there exists an $\widehat{x} \in K$ such that $P(\widehat{x})=\emptyset$.

\section{Random Equilibria of Random Games}

As an application of our random fixed point theorem, namely, Theorem A above, we shall first prove the following existence theorem of random maximal elements:

Theorem 1. Let $(\Omega, \Sigma)$ be a measurable space, $\Sigma$ Suslin family, $X$ a non-empty paracompact convex and Suslin subset of a Hausdorff topological vector space $E$ and $Q: \Omega \times X \rightarrow 2^{X}$ such that for each given $\omega \in \Omega, Q(\omega, \cdot)$ is $L_{I_{X}}$-majorized and DomQ $\in \Sigma \otimes \mathfrak{B}(X)$. Suppose that for each fixed $\omega \in \Omega$, there exists a non-empty compact convex subset $X_{0}(\omega)$ of $X$ and a non-empty compact subset $K(\omega)$ of $X$ such that for each $y \in X \backslash K(\omega)$ there is an $x \in \operatorname{co}\left(X_{0}(\omega) \cup\{y\}\right)$ with $x \in \operatorname{co} Q(\omega, y)$. Then $Q$ has a random maximal element, i.e., there exists a measurable mapping $\psi: \Omega \rightarrow X$ such that $Q(\omega, \psi(\omega))=\emptyset$ for all $\omega \in \Omega$.

Proof. By Lemma 3, for each $\omega \in \Omega$, there exists $x_{\omega} \in X$ such that $Q\left(\omega, x_{\omega}\right)=\emptyset$. Define $F: \Omega \times X \rightarrow 2^{X}$ by $F(\omega, x)=\{y \in X: Q(\omega, y)=\emptyset\}$ for each $(\omega, x) \in \Omega \times X$. Then for each fixed $\omega \in \Omega, x_{\omega}$ is a fixed point of $F(\omega, \cdot)$. In order to prove that $\operatorname{Graph} F \in \Sigma \otimes \mathfrak{B}(X \times X)$, we define a mapping $C: \Omega \times X \times X \rightarrow \Omega \times X \times X$ by

$$
C(\omega, x, y)=(\omega, y, x)
$$

for each $(\omega, x, y) \in \Omega \times X \times X$. Then $C$ is measurable. By hypothesis, $D o m Q \in \Sigma \otimes \mathfrak{B}(X)$. Since

$$
\operatorname{GraphF}=\{(\omega, x, y) \in \Omega \times X \times X: Q(\omega, y)=\emptyset\}
$$




$$
=C^{-1}[(\Omega \times X \backslash D o m Q) \otimes X] \in \Sigma \otimes \mathfrak{B}(X \times X),
$$

then, by Theorem A, $F$ has a random fixed point $\psi$, i.e., there exists $\psi: \Omega \rightarrow X$ is measurable such that $\psi(\omega) \in F(\omega, \psi(\omega))$ for all $\omega \in \Omega$ which imp! s that $Q(\omega, \psi(\omega))=\emptyset$ for each $\omega \in \Omega$.

As an application of Theorem A again, we have the following existence theorem of random equilibria for random one-person games:

Theorem 2. Let $(\Omega, \Sigma)$ be a measurable space, $\Sigma$ a Suslin family and $X$ a non-empty precompact convex and Suslin subset of a Hausdorff topological vector space. Let $A, B, P$ : $\Omega \times X \rightarrow 2^{X}$ be such that

(i) for each $\omega \in \Omega, A(\omega, \cdot) \cap P(\omega, \cdot)$ is L-majorized;

(ii) $\quad A(\omega, x)$ is non-empty and $\operatorname{co} A(\omega, x) \subset B(\omega, x)$ for each $(\omega, x) \in \Omega \times X$;

(iii) $\quad(A(\omega, \cdot))^{-1}(y)=\{x \in X: y \in A(\omega, x)\}$ is open in $X$ for each $(\omega, y) \in \Omega \times X$;

(iv) $\operatorname{Dom}(A \cap P)$ and $\operatorname{Proj}_{\Omega \times X}[(\operatorname{Graph} \bar{B}) \cap(\Omega \times \Delta)] \in \Sigma \otimes \mathscr{B}(X)$ where $\Delta=\{(x, x)$ : $x \in X\}$;

(v) for each fixed $\omega \in \Omega$, there exist a non-empty compact convex subset $X_{0}(\omega)$ of $X$ and a non-empty compact subset $K(\omega)$ of $X$ such that for each $y \in X \backslash K(\omega)$ there is an $x \in \operatorname{co}\left(X_{0}(\omega) \cap\{y\}\right)$ with $x \in \operatorname{co}(P(\omega, y) \cap A(\omega, y))$.

Then the random one-person game $(\Omega ; X ; A, B ; P)$ has a random equilibrium, i.e, there exists a measurable mapping $\psi: \Omega \rightarrow X$ such that $\psi(\omega) \in \bar{B}(\omega, \psi(\omega))$ and $A(\omega, \psi(\omega)) \cap P(\omega, \psi(\omega))=\emptyset$ for all $\omega \in \Omega$.

Proof. Define $\Psi: \Omega \times X \rightarrow 2^{X}$ by

$$
\Psi(\omega, x)=\{y \in X: A(\omega, y) \cap P(\omega, y)=\emptyset \text { and } y \in \bar{B}(\omega, y)\}
$$

for each $(\omega, x) \in \Omega \times X$. Then by Theorem 2 of Ding and Tan [11], for each $\omega \in \Omega$, there exists $x_{\omega} \in X$ such that $x_{\omega} \in \Psi(\omega, x)$ for all $x \in X$. It follows that $\Psi: \Omega \times X \rightarrow 2^{X} \backslash\{\emptyset\}$ and $x_{\omega} \in \Psi\left(\omega, x_{\omega}\right)$ for all $\omega \in \Omega$ so that $\Psi$ has a deterministic fixed point in $X$. Now define a mapping $C: \Omega \times X \times$ $X \rightarrow \Omega \times X \times X$, by $C(\omega, x, y)=(\omega, y, x)$ for each $(\omega, x, y) \in \Omega \times X \times X$. Then $C$ is measurable. Note that

$$
\begin{aligned}
\text { Graph } \Psi= & \left.C^{-1}([\Omega \times X) \backslash \operatorname{Dom}(A \cap P)] \times X\right) \\
& \cap C^{-1}\left(\operatorname{Proj}_{\Omega \times X}[\operatorname{Graph} \bar{B} \cap(\Omega \times \Delta)] \times X\right) \\
& \in \Sigma \otimes \mathscr{B}(X \times X),
\end{aligned}
$$

so that $\operatorname{Graph} \Psi \in \Sigma \otimes \mathscr{B}(X \times X)$. By Theorem A, $\Psi$ has a random fixed point $\psi$, i.e., $\psi: \Omega \times X$ is measurable such that $A(\omega, \psi(\omega)) \cap P(\omega, \psi(\omega))=\emptyset$ and $\psi(\omega) \in \bar{B}(\omega, \psi(\omega))$ for all $\omega \in \Omega$.

As another application of Theorem A, we have the following:

Theorem 3. Let $(\Omega, \Sigma)$ be a measurable space with $\Sigma$ a Suslin family and $\Gamma=\left(\Omega ; X_{i} ; A_{i}, B_{i}\right.$; $\left.P_{i}\right)_{i \in I}$ a random generalized game such that $I$ is countable and $X=\Pi_{i \in I} X_{i}$ is paracompact. For each $i \in I$, suppose that the following conditions are satisfied:

(I) $X_{i}$ is a non-empty convex and Susin subset of a locally convex Hausdorff topological vector space;

(II) $\operatorname{Dom}\left(A_{i} \cap P_{i}\right), \operatorname{Proj}_{\Omega \times X}\left[\operatorname{Graph} \overline{B_{i}} \cap\left(\Omega \times \Delta_{i}\right)\right] \in \Sigma \otimes \mathscr{B}(X)$ where $\Delta_{i}=\left\{\left(x, \pi_{i}(x)\right)\right.$ : $x \in X\}$.

(III) for each $\omega \in \Omega, E_{i}(\omega)=\left\{x \in X: A_{i}(\omega, x) \cap P_{i}(\omega, x) \neq \emptyset\right\}$ is open in $X$;

(IV) for each fixed $\omega \in \Omega$, either

(i) (a) $A_{i}(\omega, \cdot): X \rightarrow 2^{X_{i}}$ is lower semicontinuous such that for each $x \in X$, $A_{i}(\omega, x)$ is non-empty and $c o A_{i}(\omega, x) \subset B_{i}(\omega, x)$, and 
or

(b) $A_{i}(\omega, \cdot) \cap P_{i}(\omega, \cdot)$ is L-majorized;

(ii) (a) $B_{i}(\omega, \cdot)$ is upper semicontinuous with non-empty compact and convex values such that for each $x \in X, A_{i}(\omega, x) \subset B_{i}(\omega, x)$, and

(b) $P_{i}(\omega, \cdot)$ is lower semicontinuous and L-majorized, and $X_{i}$ is closed and has the property $(K)$;

$(V)$ for each fixed $\omega \in \Omega$, there exist a non-empty compact convex subset $X_{0}(\omega)$ of $X$ and a non-empty compact subset $K(\omega)$ of $X$ such that for each $y \in X \backslash K(\omega)$ there is an $x \in \operatorname{co}\left(X_{0}(\omega) \cup\{y\}\right)$ with $x_{i} \in \operatorname{co}\left(A_{i}(\omega, y) \cap P_{i}(\omega, y)\right)$ for all $i \in I$.

Then $\Gamma$ as a random equilibrium.

Proof. First we note that as each $X_{i}$ is a Suslin space and $I$ is countable, $X$ is also a Suslin space. For each $i \in I$, define $\Psi_{i}: \Omega \times X \rightarrow 2^{X}$ by

$$
\Psi_{i}(\omega, x)=\left\{y \in X: A_{i}(\omega, y) \cap P_{i}(\omega, y)=\emptyset \text { and } \pi_{i}(y) \in \overline{B_{i}}(\omega, y)\right\}
$$

for each $(\omega, x) \in \Omega \times X$. Define $\Psi: \Omega \times X \rightarrow 2^{X}$ by $\Psi(\omega, x)=\bigcap_{i \in I} \Psi_{i}(\omega, x)$ for each $(\omega, x) \in \Omega \times X$. Then by Lemma 1 or Lemma 2 , for each $\omega \in \Omega$, there exists $x_{\omega} \in X$ such that $x_{\omega} \in \Psi_{i}(\omega, x)$ for all $x \in X$ and for all $i \in I$ so that $x_{\omega} \in \Psi(\omega, x)$ for all $x \in X$. It follows that $\Psi: \Omega \times X \rightarrow 2^{X} \backslash\{\emptyset\}$ and $x_{\omega} \in \Psi\left(\omega, x_{\omega}\right)$ for all $\omega \in \Omega$ so that $\Psi$ has a deterministic fixed point in $X$. Now define a mapping $C: \Omega \times X \times X \rightarrow \Omega \times X \times X$ by

$$
C(\omega, x, y)=(\omega, y, x)
$$

for each $(\omega, x, y) \in \Omega \times X \times X$. Then $C$ is measurable. Note that

$$
\begin{aligned}
\operatorname{Graph}_{i}= & C^{-1}\left(\left[\Omega \times X \backslash \operatorname{Dom}\left(A_{i} \cap P_{i}\right)\right] \times X\right) \\
& \cap C^{-1}\left(\operatorname{Proj}_{\Omega \times X}\left[\operatorname{Graph} \overline{B_{i}} \cap\left(\Omega \times \Delta_{i}\right)\right] \times X\right) \\
& \in \Sigma \otimes \mathscr{B}(X \times X),
\end{aligned}
$$

and $I$ is countable, we have $G r a p h \Psi=\bigcap_{i \in I} \operatorname{Graph} \Psi_{i} \in \Sigma \otimes \mathscr{B}(X \times X)$. By Theorem A, there exists a measurable mapping $\psi: \Omega \rightarrow X$ such that $\psi(\omega) \in \Psi(\omega, \psi(\omega))$ for all $\omega \in \Omega$; i.e., $A_{i}\left(\omega, \psi(\omega) \cap P_{i}(\omega, \psi(\omega))=\emptyset\right.$ and $\pi_{i}\left(\psi_{i}(\omega)\right) \in \overline{B_{i}}(\omega, \psi(\omega))$ for all $\omega \in \Omega$ and for all $i \in I$.

As a consequence of Theorem 3, we have the following existence theorem of random qualitative games:

Theorem 4. Let $(\Omega, \Sigma)$ be a measurable space with $\Sigma$ a Suslin family and $\Gamma=\left(\Omega ; X_{i} ; P_{i}\right)_{i \in I}$ a random qualitative game such that $I$ is countable and $X=\Pi_{i \in I} X_{i}$ is paracompact. For each $i \in I$, suppose that the following conditions are satisfied:

(i) $\quad X_{i}$ is a non-empty convex and Suslin subset of a locally convex Hausdorff topological vector space;

(ii) $\quad \operatorname{DomP}_{i} \in \Sigma \otimes \mathfrak{B}(X)$;

(iii) for each $\omega \in \Omega, \operatorname{DomP}_{i}(\omega, \cdot)$ is open in $X$;

(iv) for each fixed $\omega \in \Omega, P_{i}(\omega, \cdot)$ is L-majorized;

$(v) \quad$ for each fixed $\omega \in \Omega$, there exist a non-empty compact convex subset $X_{0}(\omega)$ of $X$ and a non-empty compact subset $K(\omega)$ of $X$ such that for each $y \in X \backslash K(\omega)$ there is an $x \in \operatorname{co}\left(X_{0}(\omega) \cup\{y\}\right)$ with $x_{i} \in \operatorname{co}\left(P_{i}(\omega, y)\right)$ for all $i \in I$.

Then $\Gamma$ has a random equilibrium.

Proof. For each $i \in I$, define $A_{i}, B_{i}: \Omega \times X \rightarrow 2^{X}$ by $A_{i}(\omega, x)=B_{i}(\omega, x)=X$ for each 
$(\omega, x) \in \Omega \times X$. Then it is easily seen that all hypotheses of Theorem 3 are satisfied. By Theorem 3 , the conclusion follows.

\section{Random Quasi-Variational Inequalities}

In this section, by our existence theorems of random equilibria for random generalized games, namely, Theorem 3, some existence theorems of random quasi-variational inequalities and generalized random quasi-variational inequalities are given. Our results not only generalize the results of Tan [36] and Zhang [47], but also they are the stochastic versions of corresponding results in the literatures, e.g., see Aubin [3], Aubin and Ekeland [4], Hildenbrand and Sonnenschein [15], Shih and Tan [33], Tan [35, 36], Zhang [47], Zhou and Chen [48] and the references therein.

Here we emphasize that our arguments for the existence of solutions for non-compact random quasi-variational inequalities are different from the approaches used in the literatures by Tan [36] and Zhang [47].

Theorem 5. Let $(\Omega, \Sigma)$ be a measurable space with $\Sigma$ a Suslin family and I be countable. For each $i \in I$, suppose that the following conditions are satisfied:

(a) $X_{i}$ is a non-empty convex and closed Suslin subset of a locally convex Hausdorff topological vector space such that $X_{i}$ has the property $(K)$ and $X=\Pi_{i \in I} X_{i}$ is paracompact;

(b) for each fixed $\omega \in \Omega, A_{i}(\omega, \cdot): X=\Pi_{i \in I} X_{i} \rightarrow 2 X_{i}$ is upper semicontinuous with non-empty compact and convex values;

(c) $\quad \psi_{i}: \Omega \times X \times X_{i} \rightarrow \mathbb{R} \cup\{-\infty,+\infty\}$ is such that:

$(c)_{1}: \quad x \mapsto \psi_{i}(\omega, x, y)$ is lower semicontinuous on $X$ for each fixed $(\omega, y) \in \Omega \times X_{i}$;

$(c)_{2}: \quad x_{i} \notin \operatorname{co}\left\{y \in X_{i}: \psi_{i}(\omega, x, y)>0\right\}$ for each fixed $(\omega, x) \in \Omega \times X$;

$(c)_{3}$ : for each fixed $\omega \in \Omega$, the set $\left\{x \in X: \alpha_{i}(\omega, x)>0\right\}$ is open in $X$, where $\alpha_{i}: \Omega \times$ $X \mapsto \mathbb{R} \cup\{-\infty,+\infty\}$ is defined by $\alpha_{i}(\omega, x)=\sup _{y_{i} \in A_{i}(\omega, x)} \psi_{i}\left(\omega, x, y_{i}\right)$ for each $(\omega, x) \in \Omega \times X$

(d) $\quad\left\{(\omega, x) \times X: \alpha_{i}(\omega, x)>0\right\}$, and $\left\{(\omega, x) \in \Omega \times X: \pi_{i}(x) \in A_{i}(\omega, x)\right\} \in \Sigma \otimes \mathscr{B}(X)$;

(e) for each given $\omega \in \Omega$, there exist a non-empty compact convex subset $X_{0}(\omega)$ of $X$ and a non-empty compact subset $K(\omega)$ of $X$ such that for each $y \in X \backslash K(\omega)$ there exists $x \in \operatorname{co}\left(X_{0}(\omega) \cup\{y\}\right)$ with $x_{i} \in \operatorname{co}\left(A_{i}(\omega, y) \cap\left\{z \in X_{i}: \psi_{i}(\omega, y, z)>0\right\}\right)$.

Then there exists a measurable mapping $\phi: \Omega \rightarrow X$ such that for $i \in I, \pi_{i}(\phi(\omega)) \in A_{i}(\omega, \phi(\omega))$ and

for all $\omega \in \Omega$.

$$
\sup _{y \in A_{i}(\omega, \phi(\omega))} \psi_{i}(\omega, \phi(\omega), y) \leq 0
$$

Proof. For each $i \in I$, define $P_{i}: \Omega \times X \rightarrow 2^{X_{i}}$ by $P_{i}(\omega, x)=\left\{y \in X_{i}: \psi_{i}(\omega, x, y)>0\right\}$ for each $(\omega, x) \in \Omega \times X$. We shall show that $G=\left(\Omega ; X_{i} ; A_{i} ; P_{i}\right)_{i \in I}$ satisfies all hypotheses of Theorem 3 with $A_{i}=B_{i}$ for all $i \in I$.

Suppose $i \in I$ and $\omega \in \Omega$. By $(c)_{1}$, for each fixed $y \in X_{i},\left(P_{i}(\omega, \cdot)\right)^{-1}(y)=\{x \in X$ : $\left.\psi_{i}(\omega, x, y)>0\right\}$ is open in $X$ and by $(c)_{2}, x_{i} \notin c o P_{i}(\omega, x)$ for each $x \in X$. This shows that $P_{i}(\omega, \cdot)$ is lower semicontinuous and is of class $L$ and hence is $L$-majorized. By the definition of $\alpha_{i}$, we note that $\left\{x \in X: A_{i}(\omega, x) \cap P_{i}(\omega, x) \neq \emptyset\right\}=\left\{x \in X: \alpha_{i}(\omega, x)>0\right\}$, so that $\{x \in X$ : $\left.A_{i}(\omega, x) \cap P_{i}(\omega, x) \neq \emptyset\right\}$ is open in $X$ by $(c)_{3}$. By $(d)$, we know that $\operatorname{Dom}\left(A_{i} \cap P_{i}\right) \in \Sigma \otimes \mathscr{B}(X)$ and

$$
\operatorname{Proj}_{\Omega \times X}\left[\text { GraphA } A_{i} \cap\left(\Omega \times \Delta_{i}\right)\right] \in \Sigma \otimes \mathscr{B}(X) .
$$

Thus $G=\left(\Omega, X_{i}, A_{i}, P_{i}\right)_{i \in I}$ satisfies all hypothesis of Theorem 3 with $A_{i}=B_{i}$ for each $i \in I$. By 
Theorem 3 , there exists a measurable mapping $\phi: \Omega \rightarrow X$ such that for each $i \in I$,

$$
\pi_{i}(\phi(\omega)) \in A_{i}(\omega, \phi(\omega)) \text { and } A_{i}(\omega, \phi(\omega)) \cap P_{i}(\omega, \phi(\omega))=\emptyset
$$

for all $\omega \in \Omega$, i.e.,

for all $\omega \in \Omega$.

$$
\pi_{i}(\phi(\omega)) \in A_{i}(\omega, \phi(\omega)) \operatorname{and}_{y \in A_{i}(\omega, \phi(\omega))} \phi_{i}(\omega, \phi(\omega), y) \leq 0
$$

Letting $I=\{1\}$ in Theorem 5, we have the following existence results on random quasi-variational inequalities:

Theorem 6. Let $(\Omega, \Sigma)$ be a measurable space with $\Sigma$ a Suslin family. Suppose that the following conditions are satisfied:

(a) $X$ is a non-empty closed paracompact convex and Suslin subset of a locally convex Hausdorff topological vector space, and $X$ has the property $(K)$;

(b) for each fixed $\omega \in \Omega, A(\omega, \cdot): X \rightarrow 2^{X}$ is upper semicontinuous with non-empty compact and convex values;

(c) $\quad \psi: \Omega \times X \times X \rightarrow \mathbb{R} \cup\{-\infty,+\infty\}$ is such that:

$(c)_{1} \quad x \mapsto \psi(\omega, x, y)$ is lower semicontinuous on $X$ for each fixed $(\omega, y) \in \Omega \times X$;

$(c)_{2} \quad x \notin c o\{y \in X: \psi(\omega, x, y)>0\}$ for each fixed $(\omega, x) \in \Omega \times X$;

$(c)_{3}$ for each fixed $\omega \in \Omega$, the set $\{x \in X: \alpha(\omega, x)>0\}$ is open in $X$, where $\alpha: \Omega \times X \mapsto$ $\mathbb{R} \cup\{-\infty,+\infty\}$ is defined by $\alpha(\omega, x)=\sup _{y \in A(\omega, x)} \psi(\omega, x, y)$ for each $(\omega, x) \in \Omega \times$ $X$;

(d) $\{(\omega, x) \in \Omega \times X: \alpha(\omega, x)>0\}$, and $\{(\omega, x) \in \Omega \times X: x \in A(\omega, x)\} \in \Sigma \otimes \mathscr{B}(X)$;

(e) for each given $\omega \in \Omega$, there exist a non-empty compact convex subset $X_{0}(\omega)$ of $X$ and a non-empty compact subset $K(\omega)$ of $X$ such that for each $y \in X \backslash K(\omega)$ there exist $x \in \operatorname{co}\left(X_{0}(\omega) \cup\{y\}\right)$ with $x \in \operatorname{co}(A(\omega, y) \cap\{z \in X: \psi(\omega, y, z)>0\})$.

Then there exists a measurable mapping $\phi: \Omega \rightarrow X$ such that $\phi(\omega) \in A(\omega, \phi(\omega))$ and

$$
\sup _{y \in A(\omega, \phi(\omega))} \psi(\omega, \phi(\omega), y) \leq 0
$$

for all $\omega \in \Omega$.

\section{Generalized Random Quasi-Variational Inequalities}

Let $(\Omega, \Sigma)$ be a measurable space, $X$ a non-empty compact convex subset of a locally convex Hausdorff topological vector $E$ and $E^{*}$ the dual space of $E$. Suppose the correspondences $F: \Omega \times X \rightarrow 2^{X}, T: \Omega \times X \rightarrow 2^{E^{*}}$ and the function $f: \Omega \times X \times X \rightarrow \mathbb{R} \cup\{-\infty,+\infty\}$ are given. We want to find a measurable mapping $\psi: \Omega \rightarrow X$ which satisfies the following generalized random quasi-variational inequalities:

$$
\left\{\begin{array}{l}
\psi(\omega) \in F(\omega, \psi(\omega)) \\
\quad \sup _{y \in F(\omega, \psi(\omega))}\left[\sup _{u \in T(\omega, \psi(\omega))} R e\langle u, \psi(\omega)-y\rangle+f(\omega, \psi(\omega, y)] \leq 0\right.
\end{array}\right.
$$

for each $\omega \in \Omega$. We also want to find two measurable maps $\psi: \Omega \rightarrow X$ and $\phi: \Omega \rightarrow E^{*}$ such that

$$
\left\{\begin{array}{c}
\psi(\omega) \in F(\omega, \psi(\omega)) \text { and } \phi(\omega) \in T(\omega, \psi(\omega)) \\
\operatorname{Re}\langle\phi(\omega), \psi(\omega)-y\rangle+f(\omega, \psi(\omega), y) \leq 0
\end{array}\right.
$$


for all $y \in F(\omega, \psi(\omega))$ and for all $\omega \in \Omega$.

In this section, by applying results in Section 3, we shall consider the generalized random variational inequality problems $(*)$ and $(* *)$ above.

Now we recall some definitions (e.g., see [48]). Let $X$ be a non-empty convex subset of topological vector space $E$. A function $\psi(\omega, y): X \times X \rightarrow \mathbb{R} \cup\{-\infty,+\infty\}$ is said to be

(1) $\gamma$-diagonally quasi-convex (respectively, $\gamma$-diagonally quasi-concave) in $y$, in short $\gamma$ - DQCX (respectively, $\gamma$-DQCV) in $y$, if for each $A \in \mathscr{F}(X)$ and each $y \in \operatorname{co}(A)$, $\gamma \leq \max _{x \in A} \psi(y, x)$ (respectively, $\gamma \geq i n f_{x \in A} \psi(y, x)$ );

(2) $\gamma$-diagonally convex (respectively, $\gamma$-diagonally concave) in $y$, in short $\gamma$-DCX (respectively, $\gamma$-DCV) in $y$, if for each $A \in \mathscr{F}(X)$ and each $y \in \operatorname{co}(A)$ with $y=\Sigma_{i=1}^{m} \lambda_{i} y_{i}\left(\lambda_{i} \geq 0\right.$, and $\left.\Sigma_{i=1}^{m} \lambda_{i}=1\right)$, we have $\gamma \leq \Sigma_{i=1}^{m} \lambda_{i} \psi\left(y, y_{i}\right)$ (respectively, $\left.\gamma \geq \Sigma_{i=1}^{m} \lambda_{i} \psi\left(y, y_{i}\right)\right)$.

Let $X$ and $Y$ be two non-empty convex subsets of $E$, we also recall that a function $\psi: X \times Y \rightarrow \mathbb{R} \cup\{-\infty,+\infty\}$ is quasi-convex (respectively, quasi-concave) in $y$, if for each fixed $x \in X$, for each $A \in \mathscr{F}(Y)$ and each $y \in \operatorname{co}(A), \psi(x, y) \leq \max _{z \in A} \psi(x, z)$ (respectively, $\psi(x, y) \geq$ $\left.\min _{z \in A} \psi(x, z)\right)$. Moreover, it is easy to verify that

$(i)$ if $\psi(x, y)$ is $\gamma$-DCX (respectively, $\gamma$-DCV) in $y$, then $\psi(x, y)$ is $\gamma$-DQCX (respectively, $\gamma$-DQCV) in $y$,

(ii) if $\psi_{i}: X \times Y \rightarrow \mathbb{R}$ is $\gamma$-DCX (respectively, $\gamma$-DCV) in $y$ for each $i=1,2, \ldots, m$, then $\psi(x, y)=\Sigma_{i=1}^{m} a_{i}(x) \psi_{i}(x, y)$ is also $\gamma$-DCX (respectively, $\gamma$-DCV) in $y$, where $a_{i}: X \rightarrow \mathbb{R}$ with $a_{i}(x) \geq 0$ and $\Sigma_{i=1}^{m} a_{i}(x)=1$ for each $x \in X$, and

(iii) the function $\psi(x, y): X \times X \rightarrow \mathbb{R} \cup\{-\infty,+\infty\}$ is 0 -DQCV in $y$ if and only if $x \notin$ $\operatorname{co}(\{y \in X: \psi(x, y)>0\})$ for each $x \in X$.

In what follows, we first consider the existence of solutions of problem $(*)$ for which monotonicity is needed.

Theorem 7. Let $(\Omega, \Sigma)$ be a measurable space with $\Sigma$ a Suslin family and $X$ a non-empty closed paracompact convex and Suslin subset of a locally convex Hausdorff topological vector space $E$ such that $X$ has the property $(K)$. Suppose that the following conditions are satisfied:

(i) $\quad F: \Omega \times X \rightarrow 2^{X}$ is such that for each fixed $\omega \in \Omega, F(\omega, \cdot)$ is upper semicontinuous with non-empty compact and convex values;

(ii) $T: \Omega \times X \rightarrow 2^{E^{*}}$ is such that for each fixed $\omega \in \Omega, T(\omega, \cdot)$ is monotone (i.e., $R e\langle u-v, y-x\rangle \geq 0$ for all $u \in T(\omega, y)$ and $v \in T(\omega, x)$ for all $x, y \in X)$ with nonempty values and for each one-dimensional flat $L \subset E,\left.T(\omega, \cdot)\right|_{L \cap X}$ is lower semicontinuous from the relative topology of $X$ into the weak*-topology $\sigma\left(E^{*}, E\right)$ of $E^{*}$;

(iii) $f: \Omega \times X \times X \rightarrow \mathbb{R} \cup\{-\infty,+\infty\}$ is such that $x \mapsto f(\omega, x, y)$ is lower semicontinuous on $X$ for each fixed $(\omega, y) \in \Omega \times X$ and for each fixed $(\omega, x) \in \Omega \times X, y \mapsto f(\omega, x, y)$ is concave and $f(\omega, x, x)=0$ for each $(\omega, x) \in \Omega \times X$;

(iv) for each fixed $\omega \in \Omega$, the set $\left\{x \in X: \sup _{y \in F(\omega, x)}\left[\sup _{u \in T(\omega, y)} \operatorname{Re}\langle u, x-y\rangle+\right.\right.$ $f(\omega, x, y)]>0\}$ is open in $X$;

(v) $\quad\left\{(\omega, x) \in \Omega \times X: \sup _{y \in F(\omega x)}\left[\sup _{u \in T(\omega, y)} \operatorname{Re}\langle u, x-y\rangle+f(\omega, x, y)\right]>0\right\} \in$ $\Sigma \otimes \mathfrak{B}(X)$

(vi) $\quad\{(\omega, x) \in \Omega \times X: x \in F(\omega, x)\} \in \Sigma \otimes \mathscr{B}(X)$;

(vii) for each given $\omega \in \Omega$, there exist a non-empty compact convex subset $X_{0}(\omega)$ of $X$ and a non-empty compact subset $K(\omega)$ of $X$ such that for each $x \in X \backslash K(\omega)$ there exists $y \in \operatorname{co}\left(X_{0}(\omega) \cup\{x\}\right)$ with $y \in \operatorname{co}\left(F(\omega, x) \cap\left\{z \in X: \sup _{u \in T(\omega, z)} \operatorname{Re}\langle u, x-z\rangle+\right.\right.$ $f(\omega, x, z)>0\})$.

Then there exists a measurable mapping $\phi: \Omega \rightarrow X$ such that $\phi(\omega) \in F(\omega, \phi(\omega))$ and 


$$
\sup _{u \in T(\omega, \phi(\omega))}[\operatorname{Re}\langle u, \phi(\omega)-y)+f(\omega, \phi(\omega), y\rangle] \leq 0
$$

for all $y \in F(\omega, \phi(\omega))$ and $\omega \in \Omega$.

Proof. Define a function $\psi: \Omega \times X \times X \rightarrow \mathbb{R} \cup\{-\infty,+\infty\}$ by

$$
\psi(\omega, x, y)=\sup _{u \in T(\omega, y)} R e\langle u, x-y\rangle+f(\omega, x, y)
$$

for each $(\omega, x, y) \in \Omega \times X \times X$. By $(i i i), x \mapsto \psi(\omega, x, y)$ is lower semicontinuous on $X$ for each $(\omega, y) \in \Omega \times X$. For each $\omega \in \Omega$, since $T(\omega, \cdot)$ is monotone, by (iii), it is easy to verify that $\psi(\omega, x, y)$ is 0 -DCV in $y$ by Proposition 3.2 of Zhou and Chen [48]. The conditions $(i)-(v i)$ imply that all hypotheses of Theorem 6 are satisfied. By Theorem 6 , there exists a measurable mapping $\Phi: \Omega \rightarrow X$ such that $\phi(\omega) \in F(\omega, \phi(\omega))$ and

$$
\sup _{y \in F(\omega, \phi(\omega))} \sup _{u \in T(\omega, y)}[\operatorname{Re}\langle u, \phi(\omega)-y\rangle+(\omega, \phi(\omega), y)] \leq 0
$$

for all $\omega \in \Omega$. We shall now prove that

$$
\sup _{y \in F(\omega, \phi(\omega))} \sup _{u \in T(\omega, \phi(\omega))}[\operatorname{Re}\langle u, \phi(\omega)-y\rangle+f(\omega, \phi(\omega), y)] \leq 0
$$

for each $\omega \in \Omega$.

Fix an $\omega \in \Omega$. Let $x \in F(\omega, \phi(\omega))$ be arbitrarily given and let $z_{t}(\omega)=t x+(1-t) \phi(\omega)=$ $\phi(\omega)-t(\phi(\omega)-x)$ for $t \in[0,1]$. As $F(\omega, \phi(\omega))$ is convex, we have $z_{t}(\omega) \in F(\omega, \phi(\omega))$ for $t \in[0,1]$. Therefore, by (1) we have

for all $t \in[0,1]$.

$$
\sup _{u \in T\left(\omega, z_{t}(\omega)\right)}\left[\operatorname{Re}\left\langle u, \phi(\omega)-z_{t}(\omega)\right\rangle+f\left(\omega, \phi(\omega), z_{t}(\omega)\right)\right] \leq 0
$$

Since for each $x \in X, y \mapsto f(\omega, x, y)$ is concave and $f(\omega, x, x)=0$, it follows that for $t \in(0,1]$,

$$
\begin{gathered}
\quad t \cdot\left\{\sup _{u \in T\left(\omega, z_{t}(\omega)\right)}[\operatorname{Re}\langle u, \phi(\omega)-x\rangle]+f(\omega, \phi(\omega), x)\right\} \\
\leq \sup _{u \in T\left(\omega, z_{t}(\omega)\right)} t \cdot[\operatorname{Re}\langle u, \phi(\omega)-x\rangle]+f(\omega, \phi(\omega), t x+(1-t) \phi(\omega)) \\
=\sup _{u \in T\left(\omega, z_{t}(\omega)\right)}\left[\operatorname{Re}\left\langle u, \phi(\omega)-z_{t}(\omega)\right\rangle\right]+f\left(\omega, \phi(\omega), z_{t}(\omega)\right) \leq 0
\end{gathered}
$$

which implies that for $t \in(0,1]$,

$$
\left.\sup _{u \in T\left(\omega, z_{t}(\omega)\right)}[\operatorname{Re}\langle u, \phi(\omega)-x\rangle]+f(\omega, \phi(\omega), x)\right] \leq 0 .
$$

Let $z_{0} \in T(\omega, \phi(\omega))$ be arbitrarily fixed. For each $\epsilon>0$, let

$$
U_{z_{0}}=\left\{z \in E^{*}:\left|\operatorname{Re}\left\langle z_{0}-z, \phi(\omega)-x\right\rangle\right|<\epsilon\right\}
$$

Then $U_{z_{0}}$ is a $\sigma\left(E^{*}, E\right)$-neighborhood of $z_{0}$. Since $\left.T(\omega, \cdot)\right|_{L \cap X}$ is lower semicontinuous where $L:=\left\{z_{t}(\omega): t \in[0,1]\right\}$, and $U_{z_{0}} \cap T(\omega, \phi(\omega)) \neq \emptyset$, there exists a neighborhood $N(\phi(\omega))$ of $\phi(\omega)$ in $L$ such that if $z \in N(\phi(\omega))$, then $T(\omega, \phi(\omega)) \cap U_{z_{0}} \neq \emptyset$. But then there exists $\delta \in(0,1]$ such that $z_{t}(\omega) \in N(\phi(\omega))$ for all $t \in(0, \delta)$. Fixing any $t \in(0, \delta)$ and $u \in T\left(\omega, z_{t}(\omega)\right) \cap U_{z_{0}}$, we have 
$\left|R e\left\langle z_{0}-u, \phi(\omega)-z\right\rangle\right|<\epsilon$. This implies that

$$
\operatorname{Re}\left\langle z_{0}, \phi(\omega)-x\right\rangle<\operatorname{Re}\langle u, \phi(\omega)-z\rangle+\epsilon .
$$

Thus

$$
\operatorname{Re}\left\langle z_{0}, \phi(\omega)-x\right\rangle+f(\omega, \phi(\omega), x)<\operatorname{Re}\langle u, \phi(\omega)-x\rangle+f(\omega, \phi(\omega), x)+\epsilon<\epsilon
$$

by (2). Since $\epsilon>0$ is arbitrary, $R e\left\langle z_{0}, \phi(\omega)-x\right\rangle+f(\omega, \phi(\omega), x) \leq 0$. As $z_{0} \in T(\omega, \phi(\omega))$, is arbitrary, we have the following

for all $x \in F(\omega, \phi(\omega))$.

$$
\sup _{z \in T(\omega, \phi(\omega))}[\operatorname{Re}\langle z, \phi(\omega)-x\rangle+f(\omega, \phi(\omega), x)] \leq 0
$$

Corollary 8. Let $(\Omega, \Sigma)$ be a measurable space with $\Sigma$ a Suslin family, $X$ a non-empty compact convex Suslin subset of a locally convex Hausdorff topological vector space $E$ and $F: \Omega \times$ $X \rightarrow 2^{X}$ be such that $\{(\omega, x) \in \Omega \times X: x \in F(\omega, x)\} \in \Sigma \otimes \mathfrak{B}(X)$. If for each fixed $\omega \in \Omega, F(\omega, \cdot)$ is upper semicontinuous with non-empty compact convex values, then $F$ has a random fixed point.

We shall now observe that in Theorem 7 , the interaction between the correspondences $T$ and $F$ (namely, the condition $(i v)$ ) can be achieved by imposing additional continuity conditions on $T$ and $F$.

Theorem 9. Let $(\Omega, \Sigma)$ be a measurable space with $\Sigma$ a Suslin family and $X$ a non-empty closed paracompact convex and Suslin bounded subset of a locally convex Hausdorff topological vector space $E$ such that $X$ has the property $(K)$. If $F: \Omega \times X \rightarrow 2^{X}$ is such that for each $\omega \in \Omega$, $F(\omega, \cdot)$ is continuous with non-empty compact and convex values, and $T: \Omega \times X \rightarrow 2^{E^{*}}$ is such that for each $\omega \in \Omega, F(\omega, \cdot)$ is continuous with non-empty compact and convex values, and $T: \Omega \times X \rightarrow 2^{E^{*}}$ is such that for each given $\omega \in \Omega, T(\omega, \cdot)$ is monotone with non-empty values and is lower semicontinuous from the relative topology of $X$ to the strong topology of $E^{*}$. Suppose that

(i) $\quad f: \Omega \times X \times X \rightarrow \mathbb{R} \cup\{-\infty,+\infty\}$ is such that for each given $\omega \in \Omega,(x, y) \mapsto f(\omega, x, y)$ is lower semicontinuous and for each fixed $(\omega, x) \in \Omega \times X, y \mapsto f(\omega, x, y)$ is concave and $f(\omega, x, x)=0$ for each $(\omega, x) \in \Omega \times X$;

(ii) the set $\left\{(\omega, x) \in \Omega \times X: \sup _{y \in F(\omega, x)}\right.$ sup $\left._{u \in T(\omega, y)}[\operatorname{Re}\langle u, x-y\rangle+f(\omega, x, y)]>0\right\} \in$ $\Sigma \otimes \mathfrak{B}(X)$

(iii) $\quad\{(\omega, x) \in \Omega \times X: x \in F(\omega, x)\} \in \Sigma \otimes \mathfrak{B}(X)$;

(iv) for each given $\omega \in \Omega$, there exist a non-empty compact convex subset $X_{0}(\omega)$ of $X$ and a non-empty compact subset $K(\omega)$ of $X$ such that for each $x \in X \backslash K(\omega)$ there exists $y \in \operatorname{co}\left(X_{0}(\omega) \cup\{x\}\right)$ with $y \in \operatorname{co}\left(F(\omega, x) \cap\left\{z \in X: \sup _{u \in T(\omega, z)} \operatorname{Re}\langle u, x-z\rangle+\right.\right.$ $f(\omega, x, z)>0\})$.

Then there exists a measurable mapping $\phi: \Omega \rightarrow X$ such that $\phi(\omega) \in F(\omega, \phi(\omega))$ and

$$
\sup _{y \in F(\omega, \phi(\omega))}\left[\sup _{u \in T(\omega, \phi(\omega))} \operatorname{Re}\langle u \phi(\omega)-y)+f(\omega, \phi(\omega), y\rangle\right] \leq 0
$$

for all $\omega \in \Omega$.

Proof. By Theorem 7, we need only show that for each given $\omega \in \Omega$, the set

$$
\Sigma(\omega):=\left\{x \in X: \sup _{y \in F(\omega, x)}\left[\sup _{u \in T(\omega, y)} \operatorname{Re}\langle u, x-y\rangle+f(\omega, x, y)\right]>0\right\}
$$

is open in $X$. 
Since $X$ is bounded and $f(\omega, \cdot, \cdot)$ is lower semicontinuous, the function $(u, x, y) \mapsto R e\langle u, x-$ $y\rangle+f(\omega, x, y)$ is lower semicontinuous from $E^{*} \times X \times X$ to $\mathbb{R}$ for each fixed $\omega \in \Omega$. Therefore $(x, y) \mapsto \sup _{u \in T(\omega, y)}[\operatorname{Re}\langle u, x-y\rangle+f(\omega, x, y)]$ is also lower semicontinuous by lower semicontinuity of $T(\omega, \cdot)$ and Proposition III-19 of Aubin and Ekeland [4, p.118]. Since $F(\omega, \cdot)$ is lower semicontinuous, $x \mapsto s u p_{y \in F(\omega, x)^{s u p}}{ }_{u \in T(\omega, y)}[\operatorname{Re}\langle u, x-y\rangle+f(\omega, x, y)]$ is lower semicontinuous by Proposition III-19 of $[4$, p. 118] again for each fixed $\omega \in \Omega$. Thus the set $\Sigma(\omega):=\left\{x \in X: \sup _{y \in F(\omega, x)}\right.$ sup $\left._{u \in T(\omega, x)}[\operatorname{Re}\langle u, x-y\rangle+f(\omega, x, y)]>0\right\}$ is open in $X$.

Now we will consider the existence of solutions for the problems $(*)$ and $(* *)$ without assuming the monotonicity as in Theorem 9 .

Theorem 10. Let $(\Omega, \Sigma)$ be a measurable space with $\Sigma$ a Suslin family and $X$ a non-empty convex and Polish subset of a locally convex Hausdorff topological vector space E. Suppose that:

(i) $\quad F: \Omega \times X \rightarrow 2^{X}$ is such that for each $\omega \in \Omega, F(\omega, \cdot)$ is upper semicontinuous with non-empty compact and convex values;

(ii) $T: \omega \times X \rightarrow 2^{E^{*}}$ is such that $x \mapsto i n f_{u \in T(\omega, x)} R e\langle u, x-y\rangle$ is lower semicontinuous for each $(\omega, y) \in \Omega \times X$;

(iii) $f: \Omega \times X \times X \rightarrow \mathbb{R}$ is such that $x \mapsto f(\omega, x, y)$ is lower semicontinuous on $X$ for each fixed $(\omega, y) \in \Omega \times X$; and for each fixed $(\omega, x) \in \Omega \times X, y \mapsto f(\omega, x, y)$ is 0 -diagonal concave;

(iv) for each given $\omega \in \Omega$, the set

$$
\left\{x \in X: \sup _{y \in F(\omega, x)}\left[\inf _{u \in T(\omega, x)} R e\langle u, x-y\rangle+f(\omega, x, y)\right]>0\right\}
$$

is open in $X$;

(v) $\quad\left\{(\omega, x) \in \Omega \times X\right.$ sup $\left._{y \in F(\omega, x)}{ }^{i n f} f_{u \in T(\omega, x)}[R e\langle u, x-y\rangle+f(\omega, x, y)]>0\right\} \in$ $\Sigma \otimes \mathfrak{B}(X)$

(vi) $\quad\{(\omega, x) \in \Omega \times X: x \in F(\omega, x)\} \in \Sigma \otimes \mathfrak{B}(X)$;

(vii) for each $\omega \in \Omega$, there exist a non-empty compact convex subset $X_{0}(\omega)$ of $X$ and a non-empty compact subset $K(\omega)$ of $X$ such that for each $x \in X \backslash K(\omega)$ there exists $y \in \operatorname{co}\left(X_{0}(\omega) \cap\{x\}\right) \quad$ with $\quad y \in \operatorname{co}\left(F(x) \cap\left\{z \in X: \sup _{u \in T(\omega, z)} \operatorname{Re}\langle u, x-z\rangle+\right.\right.$ $f(\omega, x, z)>0\})$.

Then there exists a measurable mapping $\phi: \Omega \times X$ such that $\phi(\omega) \in F(\omega, \phi(\omega))$ and

$$
\inf _{u \in T(\omega, \phi(\omega))}[\operatorname{Re}\langle u, \phi(\omega)-y\rangle]+f(\omega, \phi(\omega), y) \leq 0
$$

for all $y \in F(\omega, \phi(\omega))$ and $\omega \in \Omega$.

Suppose that in addition,

(1) for each fixed $(\omega, x) \in \Omega \times X, y \mapsto f(\omega, x, y)$ is lower semicontinuous and concave and $f$ is measurable;

(2) there exists a non-empty Polish subset $E_{0}^{*}$ of $E^{*}$ such that $T(\Omega, X) \subset E_{0}^{*}, T$ is measurable with non-empty strongly compact convex values; and

(3) $\quad F$ is measurable.

Then there exist a measurable function $\rho: \Omega \rightarrow E^{*}$ such that $\rho(\omega) \in T(\omega, \phi(\omega))$ and

$$
\sup _{y \in F(\omega, \phi(\omega))}[\operatorname{Re}\langle\rho(\omega), \phi(\omega)-y\rangle+f(\omega, \phi(\omega), y)] \leq 0
$$

for all $\omega \in \Omega$.

Proof. Define $\psi: \Omega \times X \times X \rightarrow \mathbb{R} \cup\{-\infty,+\infty\}$ by

$$
\psi(\omega, x, y)=\inf _{u \in T(\omega, x)}[\operatorname{Re}\langle u, x-y\rangle+f(\omega, x, y)]
$$


for each $(\omega, x, y) \in \Omega \times X \times X$. Then by (ii), (iii) and (iv) we have:

(a) for each fixed $(\omega, y) \in \Omega \times X, x \mapsto \psi(\omega, x, y)$ is lower semicontinuous on $X$ and $x \notin$ $c o(\{y \in X: \psi(\omega, x, y)>0\})$ for each $(\omega, x) \in \Omega \times X$;

(b) for each $\omega \in \Omega$, the set $\left\{x \in X: \sup _{y \in F(\omega, x)} \psi(\omega, x, y)>0\right\}$ is open in $X$.

Therefore, $F$ and $\psi$ satisfy all conditions of Theorem 6 . By Theorem 6 there exists a measurable mapping $\phi: \Omega \rightarrow X$ such that $\phi(\omega) \in F(\omega, \phi(\omega))$ and

for all $\omega \in \Omega$.

$$
\sup _{y \in F(\omega, \phi(\omega))} \inf _{u \in T(\omega, \phi(\omega))}[\operatorname{Re}\langle u, \phi(\omega)-y\rangle+f(\omega, \phi(\omega), y)] \leq 0
$$

If, in addition, the conditions (1), (2) and (3) hold, we shall find another measurable (singlevalued) mapping $\rho: \Omega \rightarrow E^{*}$ such that $\rho(\omega) \in T(\omega, \phi(\omega))$ and

for each $\omega \in \Omega$.

$$
\sup _{y \in F(\omega, \phi(\omega))}[\operatorname{Re}\langle\rho(\omega), \phi(\omega)-y\rangle+f(\omega, \phi(\omega), y)] \leq 0
$$

Fix any $\omega \in \Omega$. Define $f_{1}: F(\omega, \phi(\omega)) \times T(\omega, \phi(\omega)) \rightarrow \mathbb{R}$ by

$$
f_{1}(y, u)=R e\langle u, \phi(\omega)-y\rangle+f(\omega, \phi(\omega, y)
$$

for each $(y, u) \in F(\omega, \phi(\omega)) \times T(\omega, \phi(\omega))$. Then for each $y \in F(\omega, \phi(\omega)), u \mapsto f_{1}(y, u)$ is lower semicontinuous and convex and for each fixed $u \in T(\omega, \phi(\omega)), y \mapsto f_{1}(y, u)$ is concave. By Kneser's Minimax Theorem [20],

$$
\begin{aligned}
& \inf _{u \in T(\omega, \phi(\omega))} \sup _{y \in F(\omega, \phi(\omega))}[\operatorname{Re}\langle u, \phi(\omega)-y\rangle+f(\omega, \phi(\omega), y)]= \\
& \sup _{y \in F(\omega, \phi(\omega))} \inf _{i \in T(\omega, \phi(\omega))}[\operatorname{Re}\langle u, \phi(\omega)-y\rangle+f(\omega, \phi(\omega), y)] \leq 0 .
\end{aligned}
$$

Since $T(\omega, \phi(\omega))$ is compact, there exists $u_{0} \in T(\omega, \phi(\omega))$ such that

Now we define $\Phi, T_{1}: \Omega \rightarrow 2^{X}$ by

$$
\sup _{y \in F(\omega, \phi(\omega))}\left[\operatorname{Re}\left\langle u_{0}, \phi(\omega)-y\right\rangle+f(\omega, \phi(\omega), y)\right] \leq 0 .
$$

$$
\Phi(\omega)=\left\{u \in T(\omega, \phi(\omega)): \sup _{y \in F(\omega, \phi(\omega))}[\operatorname{Re}\langle u, \phi(\omega)-y\rangle+f(\omega, \phi(\omega), y)] \leq 0\right\}
$$

and

$$
T_{1}(\omega)=T(\omega, \phi(\omega))
$$

for each $\omega \in \Omega$. Note that $\Phi(\omega) \neq \emptyset$ for all $\omega \in \Omega$. Since $T$ and $\phi$ are measurable, $T_{1}$ is also measurable by Lemma 3 in $\left[28\right.$, p. 55]. Define $g_{1}: \Omega \times X \times X \times E_{0}^{*} \rightarrow \mathbb{R}$ by

$$
g_{1}(\omega, x, y, u)=\operatorname{Re}\langle u, x-y\rangle+f(\omega, x, y)
$$

for each $(\omega, x, y, u) \in \Omega \times X \times X \times E_{0}^{*}$. Then $g_{1}$ is measurable. Also we define $g_{2}: \Omega \times X \times E_{0}^{*} \rightarrow \mathbb{R}$ by

$$
g_{2}(\omega, y, u)=R e\langle u, \phi(\omega)-y\rangle+f(\omega, \phi(\omega), y)
$$


for each $(\omega, y, u) \in \Omega \times X \times E_{0}^{*}$. Now define $F_{1}: \Omega \times 2^{X}$ by

$$
F_{1}(\omega)=F(\omega, \phi(\omega))
$$

for each $\omega \in \Omega$. Since $\phi$ is measurable and $F$ is also measurable, $g_{2}$ and $F_{1}$ are measurable by Lemma 3 in $\left[28\right.$, p. 55] again. Let $g_{3}: \Omega \times E_{0}^{*} \rightarrow \mathbb{R}$ by

$$
g_{3}(\omega, u)=\sup _{y \in F(\omega, \phi(\omega))} g_{2}(\omega, y, u)=\sup _{y \in F(\omega, \phi(\omega))}[\operatorname{Re}\langle u, \phi(\omega)-y\rangle+f(\omega, \phi(\omega), y)]
$$

for each $(\omega, u) \in \Omega \times E_{0}^{*}$. We shall show that $g_{3}$ is measurable. Since $F_{1}$ is measurable by Theorem $\mathrm{B}$, there exists a countable family of measurable mappings $p_{n}: \Omega \rightarrow X$ such that $F_{1}(\omega)=$ $c l\left\{p_{n}(\omega): n=1,2, \ldots\right\}$ for each $\omega \in \Omega$. Since $\phi$ is measurable, for each fixed $(u, y) \in E^{*} \times X$, the mapping $\omega \mapsto R e\langle u, \phi(\omega)-y\rangle$ is measurable. Note that the mapping $(u, y) \mapsto R e\langle u, \phi(\omega)-y\rangle$ is continuous, so that the mapping $(\omega, u, y) \mapsto R e\langle u, \phi(\omega)-y\rangle$ is measurable by Theorem III.14 of Castaing and Valadier [9, p. 70]. For each $n \in \mathbb{N}$, the function $g_{n}^{\prime}: \Omega \times E^{*} \rightarrow \mathbb{R}$, defined by

$$
g_{n}^{\prime}(\omega, u)=R e\left\langle u, \phi(\omega)-p_{n}(\omega)\right\rangle+f\left(\omega, \phi(\omega), p_{n}(\omega)\right)
$$

for each $(\omega, u) \in \Omega \times E^{*}$, is measurable. Therefore, for each $n \in \mathbb{N}$, the mapping $(\omega, u) \mapsto$ $\operatorname{Re}\left\langle u, \phi(\omega)-p_{n}(\omega)\right\rangle+f\left(\omega, \phi(\omega), p_{n}(\omega)\right)$ is also measurable. Since for each $(\omega, x) \in \Omega \times X$, $y \mapsto f(\omega, x, y)$ is lower semicontinuous, it follows that for each $r \in \mathbb{R}$,

$$
\left\{(\omega, u) \in \Omega \times E^{*}: g_{3}(\omega, u) \leq r\right\}=\bigcap_{n=1}^{\infty}\left\{(\omega, u) \in \Omega \times E^{*}: g_{n}^{\prime}(\omega, u) \leq r\right\} \in \Sigma \otimes \mathscr{B}\left(E^{*}\right) .
$$

Therefore the function $g_{3}$ is measurable so that the set $M_{0}=\left\{(\omega, u) \in \Omega \times E_{0}^{*}: g_{3}(\omega, u) \leq 0\right\} \in \Sigma \otimes$ $\mathscr{B}\left(E^{*}\right)$. Hence $\operatorname{Graph} \Phi=\left(\operatorname{GraphT}_{1}\right) \cap M_{0} \in \Sigma \otimes \mathscr{B}\left(E_{0}^{*}\right)$. By Theorem B, there exists a measurable mapping $\rho: \Omega \rightarrow E_{0}^{*}$ such that $\rho(\omega) \in \Phi(\omega)$ for each $\omega \in \Omega$. By the definition of $\Phi$, the measurable mapping $\rho$ satisfies the following:

$$
\left\{\begin{array}{l}
\phi(\omega) \in F(\omega, \phi(\omega)) \text { and } \rho(\omega) \in T(\omega, \phi(\omega)) \\
\sup _{y \in F(\omega, \phi(\omega))}[\operatorname{Re}\langle\rho(\omega), \phi(\omega)-y\rangle+f(\omega, \phi(\omega), y] \leq 0
\end{array}\right.
$$

Note that if $T: \Omega \times X \rightarrow 2^{E^{*}}$ is such that for each $\omega \in \Omega, T(\omega, \cdot)$ is upper semicontinuous with non-empty strongly compact values, then by Lemma 2 of Kim and Tan in [19, p. 140] or Theorem 1 of Aubin in [3, p. 67], the condition (ii) of Theorem 10 is satisfied. Thus Theorem 10 is a stochastic version of Theorem 3 of Shih and Tan in [33, p. 340]. Recall that for a topological vector space $E$, the strong topology on its dual space $E^{*}$ is the topology on $E^{*}$ generated by the family $\{U(B ; \epsilon): B$ is a non-empty bounded subset of $E$ and $\epsilon>0\}$ as a base for the neighborhood system at zero, where $U(B ; \epsilon):=\left\{f \in E^{*}: \sup _{x \in B}|\operatorname{Re}\langle f, x\rangle|<\epsilon\right\}$.

Now if we impose the upper semicontinuity condition to correspondence $T$, then we have the following:

Theorem 11. Let $(\Omega, \Sigma)$ be a measurable space with $\Sigma$ a Suslin family and $X$ a non-empty convex and Polish bounded subset of a locally convex Hausdorff topological vector space $E$. Suppose

(i) $\quad F: \Omega \times X \rightarrow 2^{X}$ is random continuous with non-empty compact and convex values;

(ii) $T: \Omega \times X \rightarrow 2^{E^{*}}$ is such that for each given $\omega \in \Omega, T(\omega, \cdot)$ is upper semicontinuous with non-empty strongly compact and convex values;

(iii) $\quad f: \Omega \times X \times X \rightarrow \mathbb{R}$ is such that

(a) for each fixed $(\omega, y) \in \Omega \times X, x \mapsto f(\omega, x, y)$ is lower semicontinuous on $X$; 
(b) for each fixed $(\omega, x) \in \Omega \times X, y \mapsto f(\omega, x, y)$ is 0 -diagonally concave;

(iv) $\quad\left\{(\omega, x) \in \Omega \times X: \sup _{y \in F(\omega, x)}\right.$ inf $\left._{u \in T(\omega, x)} R e\langle u, x-y\rangle+f(\omega, x, y)>0\right\} \in \Sigma \otimes \mathfrak{B}(X)$;

(v) for each $\omega \in \Omega$, there exist a non-empty compact convex subset $X_{0}(\omega)$ of $X$ and a non-empty compact subset $K(\omega)$ of $X$ such that for each $x \in X \backslash K(\omega)$ there exists $y \in \operatorname{co}\left(X_{0}(\omega) \cup\{x\}\right) \quad$ with $\quad y \in \operatorname{co}\left(F(x) \cap\left\{z \in X: \sup _{u \in T(\omega, z)} R e\langle u, x-z\rangle+\right.\right.$

Then, $f(\omega, x, z)>0\})$.

(a) for each fixed $\omega \in \Omega$, the set $\left\{x \in X: \sup _{y \in F(\omega, x)}{ }^{[i n f} f_{u \in T(\omega, x)} R e\langle u, x-y\rangle+\right.$ $f(\omega, x, y)]>0\}$ is open in $X$;

(b) $\quad\{(\omega, x) \in \Omega \times X: x \in F(\omega, x)\} \in \Sigma \otimes \mathscr{B}(X)$;

(c) there exists a measurable mapping $\phi: \Omega \rightarrow X$ such that $\phi(\omega) \in F(\omega, \phi(\omega))$ and

$$
i n f_{u \in T(\omega, \phi(\omega))}[\operatorname{Re}\langle u, \phi(\omega)-y\rangle+f(\omega, \phi(\omega), y)] \leq 0
$$

for all $y \in F(\omega, \phi(\omega))$ and $\omega \in \Omega$.

Proof. (a) Fix $\omega \in \Omega$. Since $X$ is a bounded subset of the locally convex Hausdorff topological vector space $E$, and $E^{*}$ is equipped with the strong topology, the function $\psi_{1}: E^{*} \times$ $X \times X \rightarrow \mathbb{R} \cup\{-\infty,+\infty\}$, defined by

$$
\psi_{1}(u, x, y)=R e\langle u, x-y\rangle
$$

for each $(u, x, y) \in E^{*} \times X \times X$, is continuous. Since $T(\omega, \cdot): X \rightarrow 2^{E^{*}}$ is upper semicontinuous with non-empty strongly compact values, by Theorem 1 of Aubin [3, p. 67], the function $\psi_{2}: X \times$ $X \rightarrow \mathbb{R} \cup\{-\infty,+\infty\}$ defined by

$$
\psi_{2}(x, y)=\inf _{u \in T(\omega, x)} R e\langle u, x-y\rangle
$$

for each $(x, y) \in X \times X$, is also lower semicontinuous. Thus the mapping $(x, y) \mapsto \inf u \in T(\omega, x)$ $R e\langle u, x-y\rangle+f(\omega, x, y)$ is lower semicontinuous by $($ iii $)$. As $F(\omega, \cdot): X \rightarrow 2^{X}$ is lower semicontinuous with non-empty values, by Proposition III-19 in [4, p. 118], the mapping $x \mapsto$ $\sup _{y \in F(\omega, x)}{ }^{i n f} f_{u \in T(\omega, x)}[\operatorname{Re}\langle u, x-y\rangle+f(\omega, x, y)]$ is lower semicontinuous from $X$ to $\mathbb{R} \cup\{-\infty,+\infty\}$ for each fixed $\omega \in \Omega$, so that the set

$$
\Sigma(\omega)=\left\{x \in X: \sup _{y \in F(\omega, x)} \inf _{u \in T(\omega, x)}[R e\langle u, x-y\rangle+(\omega, x, y)]>0\right\}
$$

is open in $X$.

(b) Since $F$ is random continuous with closed values, by Theorem 3.5 in [17, p. 57] and Lemma 2.5 of Tan and Yuan [37], the set $\{(\omega, x) \in \Omega \times X: x \in F(\omega, x)\} \in \Sigma \otimes \mathscr{B}(X)$.

Thus all hypotheses of Theorem 10 are satisfied, the conclusion follows.

If both correspondences $T$ and $F$ are measurable, we have the following:

Theorem 12. Let $(\Omega, \Sigma)$ be a measurable space with $\Sigma$ a Suslin family and $X$ a non-empty convex and Polish bounded subset of a locally convex Hausdorff topological vector space E. Suppose that

(i) $F: \Omega \times X \rightarrow 2^{X}$ is measurable such that for each $\omega \in \Omega, F(\omega, \cdot)$ is continuous with non-empty compact and convex values;

(ii) $T: \Omega \times X \rightarrow 2^{E^{*}}$ is measurable such that for each $\omega \in \Omega, T(\omega, \cdot)$ is upper semicontinuous with non-empty strongly compact and convex values; 
(iii) $\quad f: \Omega \times X \times X \rightarrow \mathbb{R}$ is measurable such that

(a) for each fixed $(\omega, y) \in \Omega \times X, x \mapsto f(\omega, x, y)$ is lower semicontinuous on $X$;

(b) for each fixed $(\omega, x) \in \Omega \times X, \quad f(\omega, x, x)=0$ and $y \mapsto f(\omega, x, y)$ is lower semicontinuous and concave;

(iv) for each $\omega \in \Omega$, there exist a non-empty compact convex subset $X_{0}(\omega)$ of $X$ and a non-empty compact subset $K(\omega)$ of $X$ such that for each $x \in X \backslash K(\omega)$ there exists $y \in \operatorname{co}\left(X_{0}(\omega) \cup\{x\}\right)$ with $y \in \operatorname{co}\left(F(\omega) \cap\left\{z \in X: s_{u} p_{u \in T(\omega, z)} R e\langle u, x-z\rangle+f(\omega, x, z)\right.\right.$ $>0\})$

Then there exist measurable maps $\phi: \Omega \rightarrow X$ and $\rho: \Omega \rightarrow E^{*}$ such that $\phi(\omega) \in F(\omega, \phi(\omega)), \rho(\omega) \in$ $T(\omega, \phi(\omega))$ and

for all $\omega \in \Omega$.

$$
\sup _{y \in F(\omega, \phi(\omega))}[\operatorname{Re}\langle\rho(\omega), \phi(\omega)-y\rangle+f(\omega, \phi(\omega), y)] \leq 0
$$

Proof. By Theorem 10 and Theorem 11, it remains to prove that $\{(\omega, x) \in \Omega \times X$ : $\sup _{y \in F(\omega, x)}$ inf $\left._{u \in T(\omega, x)} R e\langle u, x-y\rangle+f(\omega, x, y)>0\right\} \in \Sigma \otimes \mathfrak{B}(X)$.

Since $T$ and $F$ are measurable, by Theorem $4.2(e)$ of Wagner [44], there exist two countable families of measurable maps $p_{n}: \Omega \times X \rightarrow X$ and $q_{n}: \Omega \times X \rightarrow E^{*}$ such that $F(\omega, x)=\operatorname{cl}\left\{p_{n}(\omega, x)\right.$ : $n=1,2, \ldots\}$ and $T(\omega, x)=\operatorname{cl}\left\{q_{n}(\omega, x): n=1,2, \ldots\right\}$ for each $(\omega, x) \in \Omega \times X$. We define $g_{0}: E^{*} \times$ $X \times X \rightarrow \mathbb{R} \cup\{-\infty,+\infty\}$ by

$$
g_{0}(u, x, y)=R e\langle u, x-y\rangle
$$

for each $(u, x, y) \in E^{*} \times X \times X$. Then $g_{0}$ is continuous and is measurable. Therefore the function $g_{0}^{\prime}: \Omega \times E^{*} \times X \times X \rightarrow \mathbb{R} \cup\{-\infty,+\infty\}$ defined by

$$
g_{0}^{\prime}(\omega, u, x, y)=R e\langle u, x-y\rangle+f(\omega, x, y)
$$

for each $(\omega, u, x, y) \in \Omega \times E^{*} \times X \times X$, is also measurable since $f$ is measurable. Now fix any $x \in \mathbb{N}$, note that $p_{n}: \Omega \times X \rightarrow X$ is measurable and $f$ is measurable. For each $j \in \mathbb{N}$, the function $g_{j}^{n}: \Omega \times X \rightarrow \mathbb{R} \cup\{-\infty,+\infty\}$ defined by

$$
g_{j}^{n}(\omega, x)=\operatorname{Re}\left\langle q_{j}(\omega, x), x-p_{n}(\omega, x)\right\rangle+f\left(\omega, x, p_{n}(\omega, x)\right)
$$

for each $(\omega, x) \in \Omega \times X$, is measurable by Lemma 3 in $[28$, p. 55]. Therefore the mapping $g_{n}: \Omega \times X \rightarrow \mathbb{R} \cup\{-\infty,+\infty\}$ defined by

$$
g_{n}(\omega, x)=\inf _{j \in \mathbb{N}^{\prime}} g_{j}^{n}(\omega, x)=\inf _{j \in \mathbb{N}}\left[R e\left\langle q_{j}(\omega, x), x-p_{n}(\omega, x)\right\rangle+f\left(\omega, x, p_{n}(\omega, x)\right)\right]
$$

for each $(\omega, x) \in \Omega \times X$, is measurable. Note that $g: \Omega \times X \rightarrow \mathbb{R} \cup\{-\infty,+\infty\}$ defined by

$$
g(\omega, x)=\sup _{n \in \mathbb{N}} g_{n}(\omega, x)
$$

for each $(\omega, x) \in \Omega \times X$, is also measurable. Since for each $(\omega, x) \in \Omega \times X$, the mapping $y \mapsto f(\omega, x, y)$ is lower semicontinuous, the set

$$
\begin{gathered}
\left\{(\omega, x) \in \Omega \times X: \sup _{y \in F(\omega, x)} \inf _{u \in T(\omega, x)}[\operatorname{Re}\langle u, x-y\rangle+f(\omega, x, u)]>0\right\} \\
=\left\{(\omega, x) \in \Omega \times X: \sup _{n \in \mathbb{N}} \inf _{j \in \mathbb{N}}\left[\operatorname{Re}\left\langle q_{j}(\omega, x), x-p_{n}(\omega, x)\right\rangle+f\left(\omega, x, p_{n}(\omega, x)\right)\right]>0\right\} \\
=\{(\omega, x): g(\omega, x)>0\} \in \Sigma \otimes \mathscr{B}(X) .
\end{gathered}
$$


Therefore, we have

$$
\left\{(\omega, x) \in \Omega \times X: \sup _{y \in F(\omega, x)} \inf _{u \in T(\omega, x)} R e\langle u, x-y\rangle+f(\omega, x, y)>0\right\} \in \Sigma \otimes \mathscr{B}(X) .
$$

Corollary 13. Let $(\Omega, \Sigma)$ be a measurable space with $\Sigma$ a Suslin family and $X$ a non-empty compact convex subset of a Banach space $E$ whose dual space $E^{*}$ is separable. Suppose that

(i) $\quad F: \Omega \times X \rightarrow 2^{X}$ is measurable such that for each $\omega \in \Omega, F(\omega, \cdot)$ is continuous with non-empty compact and convex values;

(ii) $T: \Omega \times X \rightarrow 2^{E^{*}}$ is measurable such that for each $\omega \in \Omega, T(\omega, \cdot)$ is upper semicontinuous with non-empty strongly compact and convex values;

(iii) $\quad f: \Omega \times X \times X \rightarrow \mathbb{R}$ is measurable such that

(a) for each fixed $(\omega, y) \in \Omega \times X, x \mapsto f(\omega, x, y)$ is lower semicontinuous on $X$;

(b) for each fixed $(\omega, x) \in \Omega \times X, f(\omega, x, x)=0$ and $y \mapsto f(\omega, x, y)$ is lower semicontinuous and concave.

Then there exist measurable maps $\phi: \Omega \rightarrow X$ and $\rho: \Omega \rightarrow E^{*}$ such that $\phi(\omega) \in F(\omega, \phi(\omega)), \rho(\omega) \in$ $T(\omega, \phi(\omega))$ and

for all $\omega \in \Omega$.

$$
\sup _{y \in F(\omega, \phi(\omega))}[\operatorname{Re}\langle\rho(\omega), \phi(\omega)-y\rangle+f(\omega, \phi(\omega), y)] \leq 0
$$

By allowing $f$ to be zero in Corollary 13, we have the following:

Corollary 14. Let $(\Omega, \Sigma)$ be a measurable space with $\Sigma$ a Suslin family and $X$ a non-empty compact convex subset of a Banach space $E$ whose dual space $E^{*}$ is separable. Suppose that

(i) $\quad F: \Omega \times X \rightarrow 2^{X}$ is measurable such that for each $\omega \in \Omega, F(\omega, \cdot)$ is continuous with non-empty compact and convex values;

(ii) $T: \Omega \times X \rightarrow 2^{E^{*}}$ is measurable such that for each $\omega \in \Omega, T(\omega, \cdot)$ is upper semicontinuous with non-empty strongly compact and convex values.

Then there exist measurable map $\phi: \Omega \rightarrow X$ and $\rho: \Omega \rightarrow E^{*}$ such that $\phi(\omega) \in F(\omega, \phi(\omega))$, $\rho(\omega) \in T(\omega, \phi(\omega))$ and

for all $\omega \in \Omega$.

$$
\sup _{y \in F(\omega, \phi(\omega))} \operatorname{Re}\langle\rho(\omega), \phi(\omega)-y\rangle \leq 0
$$

Theorem 11 is also a stochastic version of Theorem 4 of Shih and Tan in [33, p. 341] (and its improvements due to Kim [18] and due to Shih and Tan [33, Theorem 2, p. 69-70] (with $M=0$ )).

Theorem 11 generalizes a theorem of Tan $[36$, p. 326] in the following ways:

(1) the correspondence $T$ is upper semicontinuous instead of being continuous, and

(2) the function $f$ need not be random continuous.

In the case where $F(x)=X$ and $T(x)=0$ for each $x \in X$, Theorem 11 also improves Theorem 9.2.3 of Zhang [47, p. 304] with weaker continuity and measurability conditions. We also remark that our arguments used in proving the existence of solutions for generalized random quasivariational inequalities in this section are different from those used by Tan [36] and Zhang [47], etc.

Quasi-variational inequalities and generalized quasi-variational inequalities have many applications in mathematical economics, game theory and optimization and other applied science (e.g., see [3-4], [7], [15] and [25]). Random quasi-variational inequalities and generalized random quasi-variational inequalities will also have many applications in random mathematical economics, random game theory and related fields. 


\section{Acknowledgement}

The author would like to express his thanks to Professors K.K. Tan and J. Dshalalow during the writing of this paper.

\section{References}

[1] Aliprantis, C., Brown, D. and Burkinshaw, O., Existence and Optimality of Competitive Equilibria, Springer-Verlag 1989.

[2] Arrow, K.J. and Debreu, G., Existence of an equilibrium for a competitive economy, Econometrica 22 (1954), 265-290.

[3] Aubin, J.P., Mathematical Methods of Game and Economics Theory (Revised Edition, ed.), North Holland 1982.

[4] Aubin, J.P. and Ekeland, I., Applied Nonlinear Analysis, John Wiley \& Sons 1984.

[5] Bharucha-Reid, A.T., Fixed point theorems in probabilistic analysis, Bull. Amer. Math. Soc. 82 (1976), 641-657.

[6] Borglin, A. and Keiding, H., Existence of equilibrium actions of equilibrium, "A note on the 'new' existence theorems", J. Math. Economics 3 (1976), 313-316.

[7] Border, K.C., Fixed Point Theorems with Applications to Economics and Game Theory, Cambridge University Press 1985.

[8] Bocsan, G., A general random fixed point theorem and application to random equations, Rev. Roum. Math. Pures et Appl. 26 (1981), 375-379.

[9] Castaing, C. and Valadier, M., Convex Analysis and Measurable Multifunctions, Lecture Notes in Math. vol. 580, Springer-Verlag 1977.

[10] Debreu, G., Existence of Competitive Equilibrium, Handbook of Mathematical Economics (ed. by K.J. Arrow and M.D. Intriligator), vol. II, North-Holland 1982, 697-743.

[11] Ding, X.P. and Tan, K.K., On equilibria of non-compact generalized games, J. Math. Anal. Appl. 177 (1993), 226-238.

[12] Ding, X.P., Kim, W.K. and Tan, K.K., Equilibria of non-compact generalized games with $\mathcal{L}^{*}$-majorized preference correspondences, J. Math. Anal. Appl. 164 (1992), 508-517.

[13] Engl, H.W., Random fixed point theorems for multivalued mappings, Pacific J. Math. 76 (1978), 351-360.

[14] Hans, O., Reduzierende zufallige transformationen, Czech. Math. J. 7 (1957), 154-158.

[15] Hildenbrand, W. and Sonnenschein, H., (eds.), Handbook of Mathematical Economics, vol. IV, North-Holland 1991.

[16] Itoh, S., Random fixed point theorem with an application to random differential equations in Banach spaces, J. Math. Anal. Appl. 67 (1979), 261-273.

[17] Himmelberg, J., Measurable relations, Fund. Math. 87 (1975), 53-72.

[18] Kim, W.K., Remark on a generalized quasi-variational inequality, Proc. Amer. Math. Soc. 103 (1988), 667-668.

[19] Kim, W.K. and Tan, K.K., A variational inequality in non-compact sets and its applications, Bull. Austral. Math. Soc. 46 (1992), 139-148.

[20] Kneser, H., Sur un théorème fondamental de la théorie des jeux, C.R. Acad. Sci. Paris 234 (1952), 2418-2420.

[21] Kucia, A. and Nowak, A., Some results and counterexamples on random fixed points, Trans. 10th Prague Conf. on Information Theory, Stat. Decision, Funct. and Random Processes Prague 1986, Academia, Prague 1988, 75-82.

[22] Leese, S.J., Multifunctions of Souslintype, Bull. Austral. Math. Soc. 11 (1974), 395-411.

[23] Lin, T.C., Random approximations and random fixed point theorems for non-self-maps, Proc. Amer. Math. Soc. 103 (1988), 1129-1135. 
[24] Liu, Z.S. and Chen, S.Z., On fixed point theorems of random set-valued maps, Kexue Tongbao (Bulletin of Chinese Science) 28 (1988), 433-435.

[25] Mas-Colell, A. and Zame, W.R., Equilibrium theory in infinite dimensional spaces, Handbook of Mathematical Economics (ed. by W. Hildenbrand and H. Sonnenschein), vol. IV (1991), 1826-1898.

[26] Nowak, A., Random fixed points of multifunctions, Prace Nauk. Uniw. Slask. Katowice, Prace Mat. 11 (1981), 36-41.

[27] Papageorgiou, N.S., Random fixed point theorems for measurable multifunctions in Banach spaces, Proc. Amer. Math. Soc. 97 (1986), 507-514.

[28] Rybinski, L.E., Random fixed points and viable random solutions of functional-differential inclusions, J. Math. Anal. Appl. 142 (1989), 53-61.

[29] Saint-Beuve, M.F., On the existence of von Neumann-Aumann's theorem, J. Funct. Anal. 17 (1974), 112-129.

[30] Saks, S., Theory of the Integral, 2nd Edition, Dover, New York 1968.

[31] Sarbadhikar, H. and Srivastava, S.M., Random versions of extension theorems of Dugundji and fixed point theorems, Boll. U.M.I. 7-B (7) (1993), 631-642.

[32] Sehgal, V.M. and Singh, S.P., On random approximations and fixed point theorems for set valued mappings, Proc. Amer. Math. Soc. 95 (1985), 91-94.

[33] Shih, M.H. and Tan, K.K., Generalized quasi-variational inequalities in locally convex topological vector spaces, J. Math. Anal. Appl. 108 (1985), 333-343.

[34] Spacek, A., Zufallige gleichungen, Czech. Math. J. 5 (1955), 462-466.

[35] Tan, K.K., Comparison theorems on minimax inequalities, variational inequalities, and fixed point theorems, J. London Math. Soc. 28 (1983), 555-562.

[36] Tan, N.X., Random quasi-variational inequality, Math. Nachr. 125 (1986), 319-328.

[37] Tan, K.K., and Yuan, X.Z., Some random fixed point theorems, Fixed Point Theory and Applications (ed. by K.K. Tan), World Scientific, Singapore 1995, 334-345.

[38] Tan, K.K. and Yuan, X.Z., On deterministic and random fixed points, Proc. Amer. Math. Soc. 119 (1993), 849-856.

[39] Tan, K.K. and Yuan, X.Z., Approximation method and equilibria of abstract economics, Proc. Amer. Math. Soc. (in press) (1994).

[40] Tan, K.K. and Yuan, X.Z., Equilibria of generalized games, Nonlinear Times and Digest 1 (1994), 61-70.

[41] Tarafdar, E. and Mehta, G., A generalized version of the Gale-Nikaido-Debreu theorem, Comm. Math. Univ: Carolinae 28 (1987), 655-659.

[42] Toussaint, S., On the existence of equilibria in economies with infinitely many commodities and without ordered preferences, J. Econ. Theory 33 (1984), 98-115.

[43] Tulcea, C.I., On the approximation of upper semicontinuous correspondences and the equilibrium of the generalized games, J. Math. Anal. Appl. 136 (1988), 267-289.

[44] Wagner, D.H., Survey of measurable selection theorems, SIAM J. Control and Optim. 15 (1977), 859-903.

[45] Xu, H.K., Some random fixed point theorems for condensing and nonexpansive operators, Proc. Amer. Math. Soc. 110 (1990), 395-400.

[46] Yannelis, N.C. and Prabhakar, N.D., Existence of maximal elements and equilibria in linear topological spaces, J. Math. Economics 12 (1983), 233-245.

[47] Zhang, S.S. (Chang), Variational Inequality and Complementarity Problem Theory with Applications, Shanghai Scientific Literature Press, Shanghai, China, 1991.

[48] Zhou, J.X. and Chen, G., Diagonal convexity conditions for problems in convex analysis and quasi-variational inequalities, J. Math. Anal. Appl. 132 (1988), 213-225. 


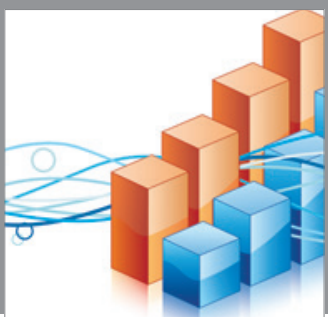

Advances in

Operations Research

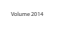

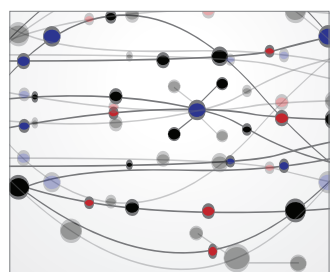

\section{The Scientific} World Journal
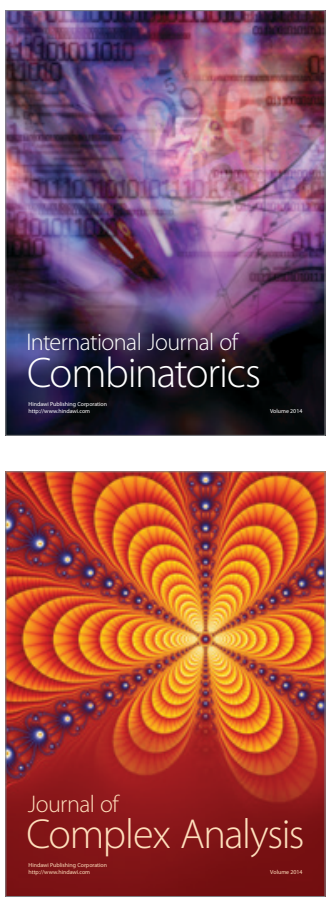

International Journal of

Mathematics and

Mathematical

Sciences
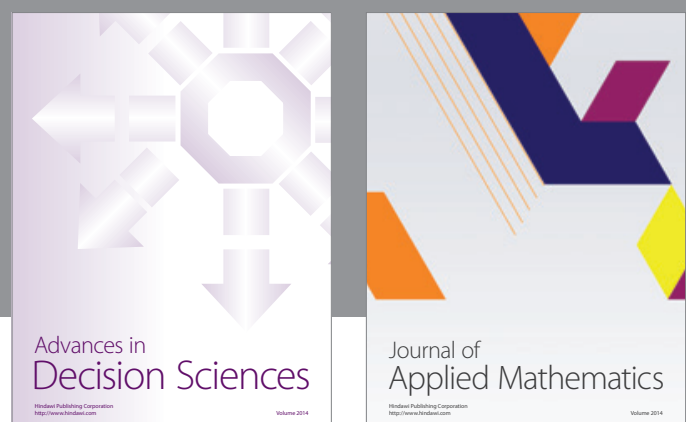

Journal of

Applied Mathematics
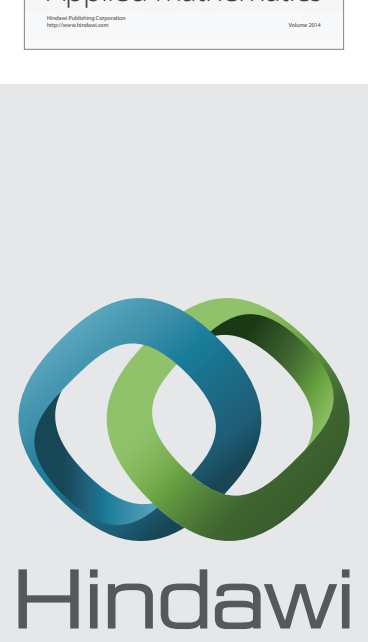

Submit your manuscripts at http://www.hindawi.com
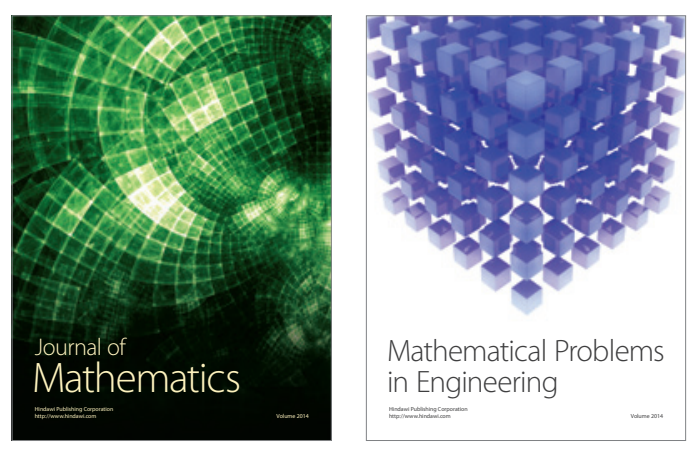

Mathematical Problems in Engineering
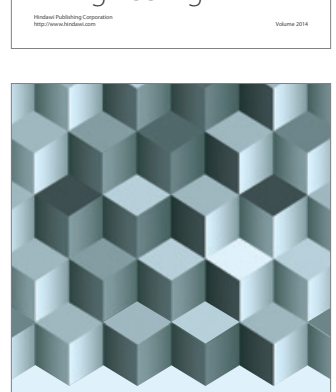

Journal of

Function Spaces
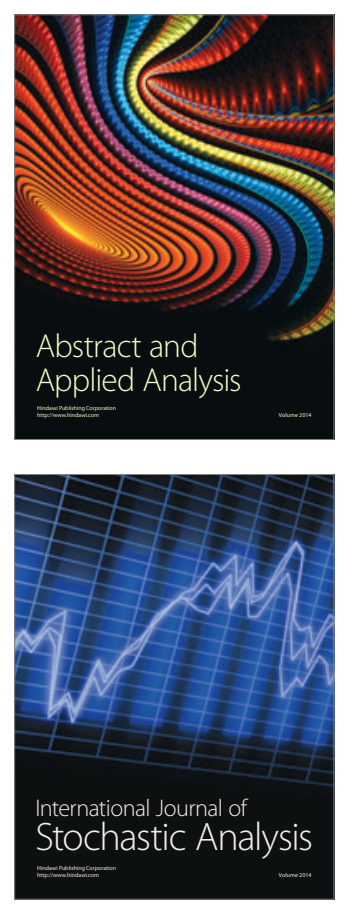

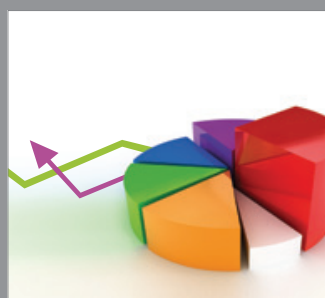

ournal of

Probability and Statistics

Promensencen
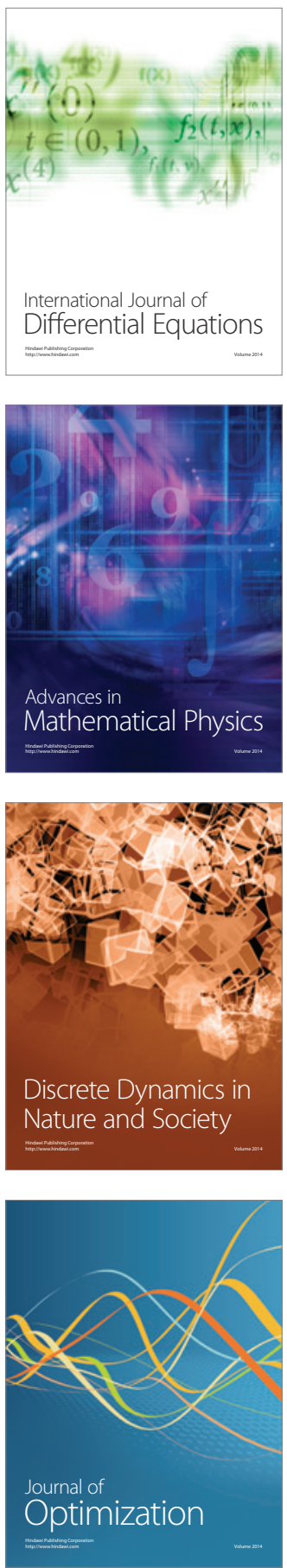